\title{
Hybridization of a hidden Markov model using Elman neural network with application
}

Omar S. Qasim

omar.saber@uomosul.edu.iq

College of Computer Science and Mathematics

University of Mosul, Mosul, Iraq

\section{Received on: 22/10/2012}

\section{ABSTRACT}

Accepted on:30 /01/2013

This research aims to improve the performance of the work of hidden Markov model, which is limited to the positive integers as input, and through the use of Elman artificial neural network that have the ability to accept all types of data in the input space. The proposed model has proved that it is highly efficient in the classification of osteoporosis data compared with Elman artificial neural network on the one hand and the hidden Markov model on the other.

Keywords: pattern recognition; Elman neural network; hidden Markov model.

تهجين أنموذج ماركوف المخفي باستخدام شبكة ايلمان العصبية الاصطناعية مع التطبيق

$$
\text { كلية علوم الحاسوب والرياضيات، جامعة الموصل قاسم }
$$

201311130 : تاريخ قبول البحث

2012110122 : تاريخ استلام البحث

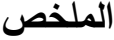

يهدف هذا البحث إلى تطوير أداء عمل أنموذج ماركوف المخفي والذي يقتصر على فضاء الإدخال من نوع الأعداد الصحيحة الموجبة, وذلك من خلال استخدام شبكة ايلمان العصبية الاصطناعية التي لها القابلية على تقبل جميع أنواع البيانات في فضـاء الإدخال. حيث اثبت الأنموذج المقترح كفاءة عالية في تصنيف بيانات هشاشـة العظام مقارنة مع شبكة ايلمان العصبية الاصطناعية من جهة وأنموذج ماركوف المخفي من جهة أخرى. الكلمات المفتاحية: تمبيز الأنعاط; الثبكة العصبية الاصطناعية ; نموذج ماركوف المخفي .

لقد أولى الباحثون في السنوات الأخيرة اهتماماً بالغاً في تصنيف الأنماط الطبية المختلفة ومن ضمنها مرض هشاشة العظام والذي يعد من الأمراض الصامتة التي تتشأ بدون ألم [8] , لذلك فأنه من الضروري جداً أن نحافظ

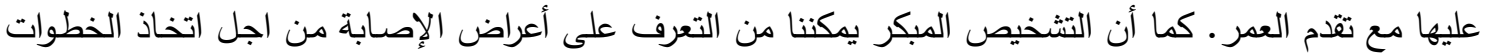
اللازمة لمنع تفاقم هذا المرض.

إن التشخيص الاقيق للبيانات يحتاج إلى اختيار متقن للنماذج المستخدمة في التعرف على الأنماط المختلفة,

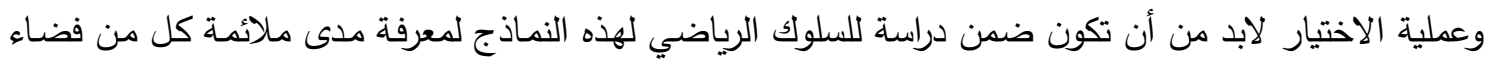

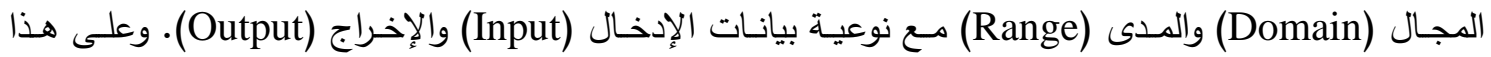
الأساس تم اختيار شبكة ايلمان العصبية كمرحلة أولى في عملية معالجة البيانات, كونها تتقبل في فضاء المجال

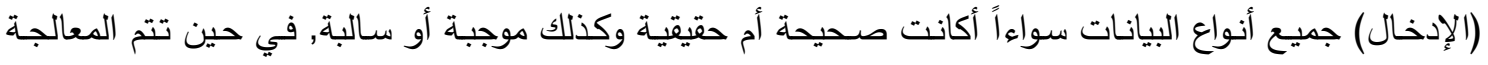
الثانية من خلال أنموذج ماركوف المخفي الذي يكون متلائماً مع طبيعة سلوك الإخراج للشبكة والذي يعطي نتائج أكثر دقة من خلال المعالجة المزدوجة. 
تم اخذ مجموعة من المشاهدات المختلفة بحجم (344) عينة, وتتكون كل عينة من (17) ميزة (متغير

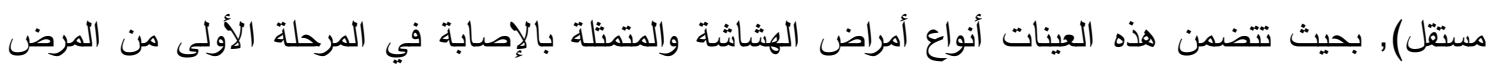

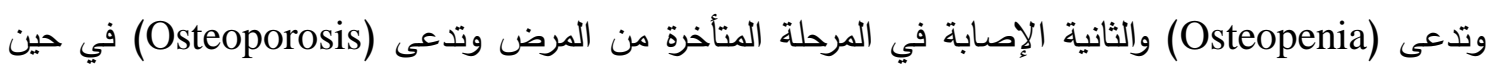

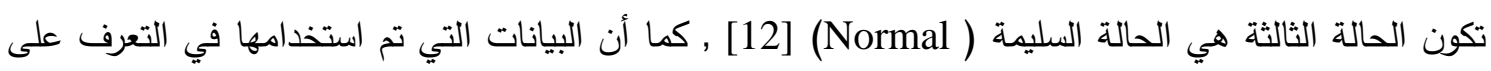
أمراض هثاشة العظام عبارة عن قيم عددية ووصفية تم الحصول عليها بمساعدة أساتذة في كلية التمريض في جامعة الموصل وتمثل معلومات ضمن قاعدة بيانات (Data Base) لأثخاص تم إجراء عملية الفحص عليهم داخل العراق وتحديداً في محافظة نينوى . إن التشخيص المتقن والتعرف الدقيق على الأنماط يعد من أهم المسائل التي تحتاج إلى اختيار دقيق لتقنيات التشخيص, وقد تم في هذا البحث دراسة سلوك كل من تقنيتي شبكة ايلمان العصبية (Elman Network) وأنموذج ماركوف المخفي (Hidden Markov) من الناحية الرياضية والتركيز على فضاءات الإدخال والإخراج والدوال الرياضية المستخدمة في عملية تحويل البيانات.

3. أنموذج ماركوف المخفي [2],[7]

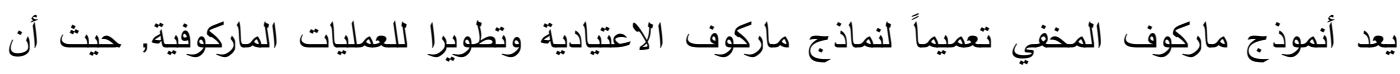
الحالات في أنموذج ماركوف الاعتيادي تكون مرئية بشكل مباشر إلى المشاهد أو المراقب, ولهذا تكون احتمالية

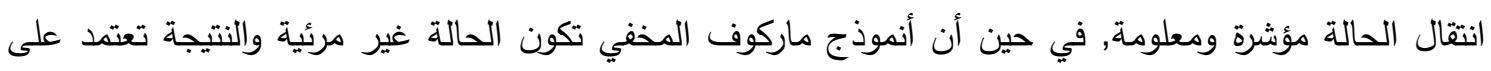

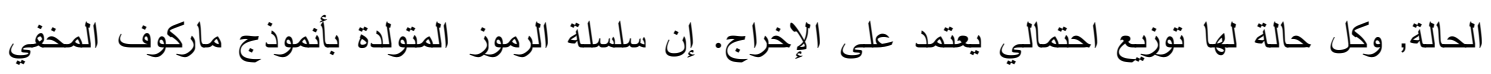
تعطي بعض المعلومات حول سلسلة الحالات.

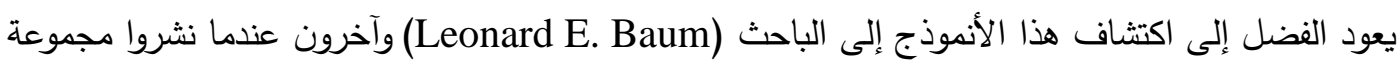

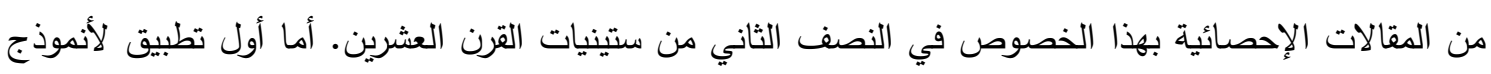
ماركوف المخفي فكان في مجال تمييز الصوت (Speech Recognition) وذلك في منتصف سبعينيات القرن العشرين. وفي النصف الثاني من ثمانينيات القرن العشرين بدأ استخدام أنموذج ماركوف المخفي في تحليل المتتابعات الحياتية (Biological Sequences) ، خصوصاً DNA، ومنذ ذلك الوقت فرض أنموذج ماركوف

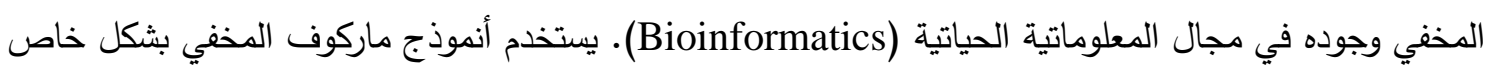
في تمييز الأنماط مثل الكلام والكتابة وكذلك في تطبيقات المعلوماتية وغيرها.

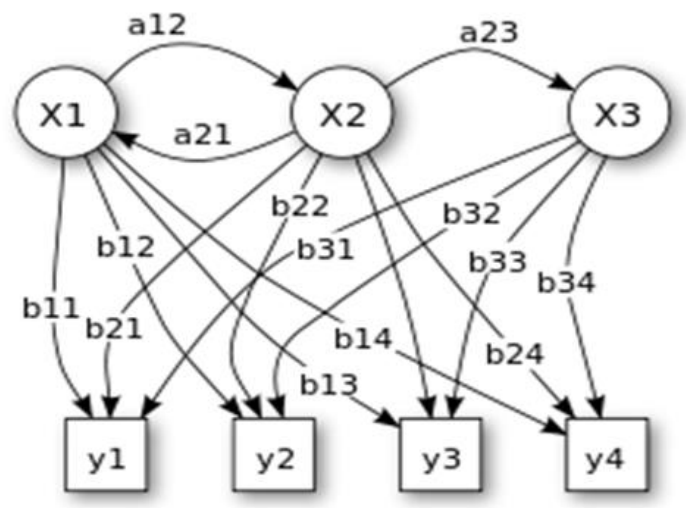

الثكل (1) يمثل المعمات الاحتمائية في أنموذج ماركوف المخفي 


$$
\begin{aligned}
& \text { في الثكل أعلاه يمكن تمثيل المعلمات بالثكل الآتي : } \\
& \text { x : تمثل الحالات (states). } \\
& \text { : تمثل المشاهدات الممكنة. } \\
& \text { a }
\end{aligned}
$$

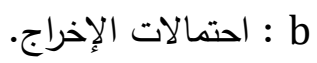

1.3

هناك ثلاث مسائل أساسية لنماذج ماركوف المخفية وهي [3]:

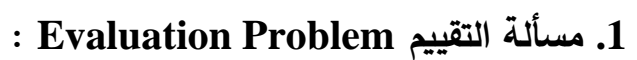

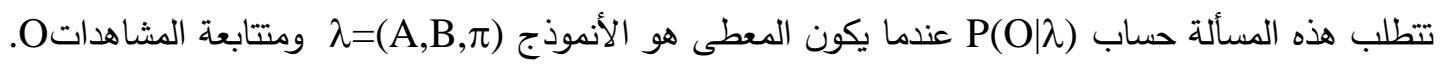

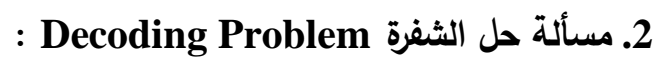

إذا كان المعطى هو الأنموذج (A,B, (A) المُثتى (Optimal) (الأكثر احتمالا).

3. Training Problem مسأكة التدريب

وتتاول هذه المسألة كيفية تعديل معلمات الأنموذج (A, B,

[6],[3] 2.3 خوارزمية فيتربي

تعمل خوارزمية (Veterbi) على إيجاد أفضل متتابعة حالة عندما يكون المعطى الأنموذج (A,

وعدد الحالات هو (N) ومتتابعة المشاهدات للخوارزمية الأمامية إلا أنها تأخذ أعلى احتمالية للمسار على احتمال المسارات السابقة, في حين أن الخوارزمية الأمامية تأخذ المجموع (Sum). كذلك فان خوارزمية فيتربي لها مؤشرات تراجعية (Back-Pointers) لاتملكها الخوارزميات الأساسية, إذ يتم حساب أفضل تسلسل للحالة عن طريق الاحتفاظ بمسار الحالات المخفية التي تقود لكل حالة, ثم تتبع أفضل مسار (Back Trace) إلى البداية. لكي يتم إيجاد متتابعة الحالة المثلى لمتتابعة المشاهدات

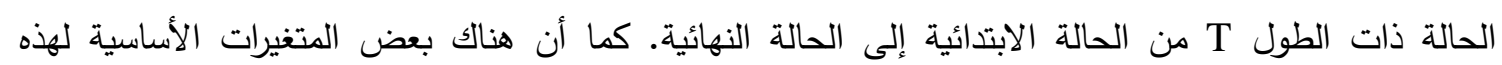
الخوارزمية وكالآتي [3]: 1. المتغير احتمالية متتابعة الحالة الجزئية الأكثر احتمالا بالنسبة لمتتابعة المشاهدات المنتهية في الحالة i ويمكن التعبير

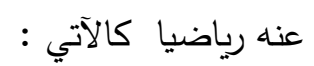
$\mathrm{V}_{\mathrm{t}}(\mathrm{j})=\max _{i=1}^{N} v_{t-1}(i) a_{i j} b_{j}\left(O_{t}\right) \quad, \mathrm{j}=1,2, \ldots, \mathrm{N} \quad, \mathrm{t}=1,2, \ldots \mathrm{T}$

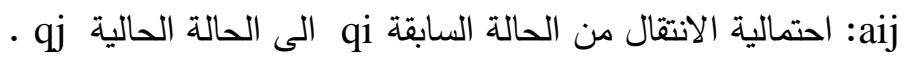

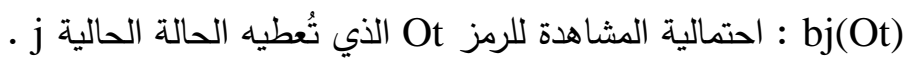
(Keep Track) للمسار الفعلي. $\psi_{t}(j)$ أما خطوات خوارزمية فيتربي فهي كما يأتي : 
2. البداية Initialization

$\mathrm{V}_{1}(\mathrm{j})=(2)$ $j=1,2, \ldots, N ; \pi_{i} b_{i}\left(O_{1}\right)$

$\psi_{1}(j)=\mathrm{O}$

$\mathrm{V}_{\mathrm{t}}(\mathrm{j})=\max _{i=1}^{N}\left[v_{t-1}(i) \cdot a_{i j}\right] b_{j}\left(O_{t}\right) \quad ; \begin{aligned} & j=1,2, \ldots, N \\ & t=2,3, \ldots, T\end{aligned}$

3. التعاقب Recursion

$\psi_{1}(j)=\underset{i=1}{N}\left[v_{t-1}(i) \cdot a_{i j}\right] \quad ; \begin{aligned} & j=1,2, \ldots, N \\ & t=2,3, \ldots, T\end{aligned}$

$\mathrm{P}^{*}=\max _{i=1}^{N}\left[v_{T}(i)\right]$

4. النهاية Termination

$\mathrm{q}^{*}=\underset{i=1}{\arg \max }\left[v_{T}(i)\right]$

$$
{ }_{i=1}
$$

$q_{T}^{*}=\psi_{t+1}\left(q_{t+1}^{*}\right) ; \mathrm{t}=\mathrm{T}-1, \mathrm{~T}-2, \ldots, 2,1$

5. التعاقب المعاكس (Back tracking)

\section{4. شبكة ايلمان العصبية الاصطناعية}

تعد شبكة ايلمان العصبية من اشهر الشبكات التكرارية التي ابتكرت من قبل العالم (Jeffrey L. Elman) في العام (1990), وهي بثكل عام نوع خاص من شبكات التغذية الخلفية التي تتكون من طبقتين تسري من فن

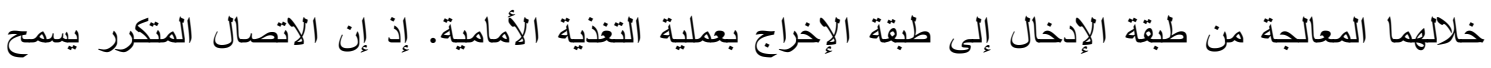

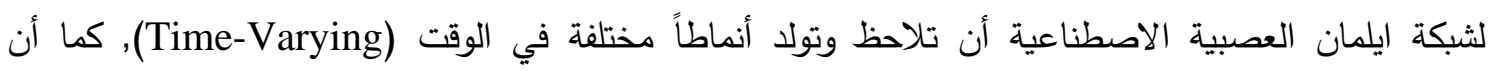

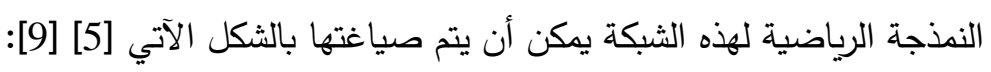

$$
\begin{aligned}
& O_{k}(t)=\sum_{j=1}^{q} w_{k j} H_{j}(t) \\
& H_{j}(t)=g\left(V_{j}(t)\right) \\
& V_{j}(t)=\sum_{a=1}^{q} w_{j a} H_{j a}(t-1)+\sum_{i=1}^{p} w_{j i} I_{i}(t)
\end{aligned}
$$

: ع عدد الوحدات العصبية في طبقة الإدخال. p

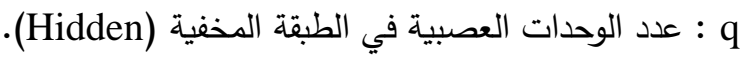

: $H_{j}(t)$ يمثل إخراج وحدات الطبقة المخفية متأخرة بمقدار وحدة زمنية واحدة.

洁 $V_{j}(t)$

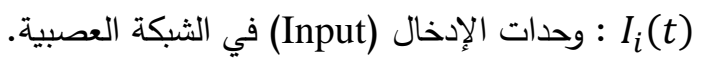

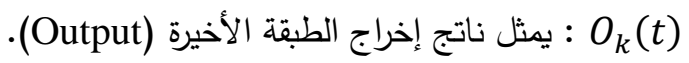

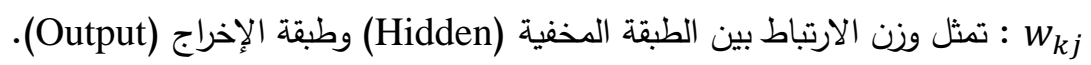
wa

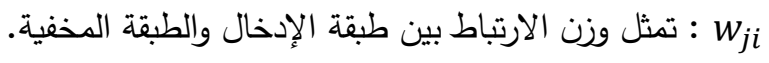
g(.) : دالة التتشيط وعادة ماتكون غير خطية. 


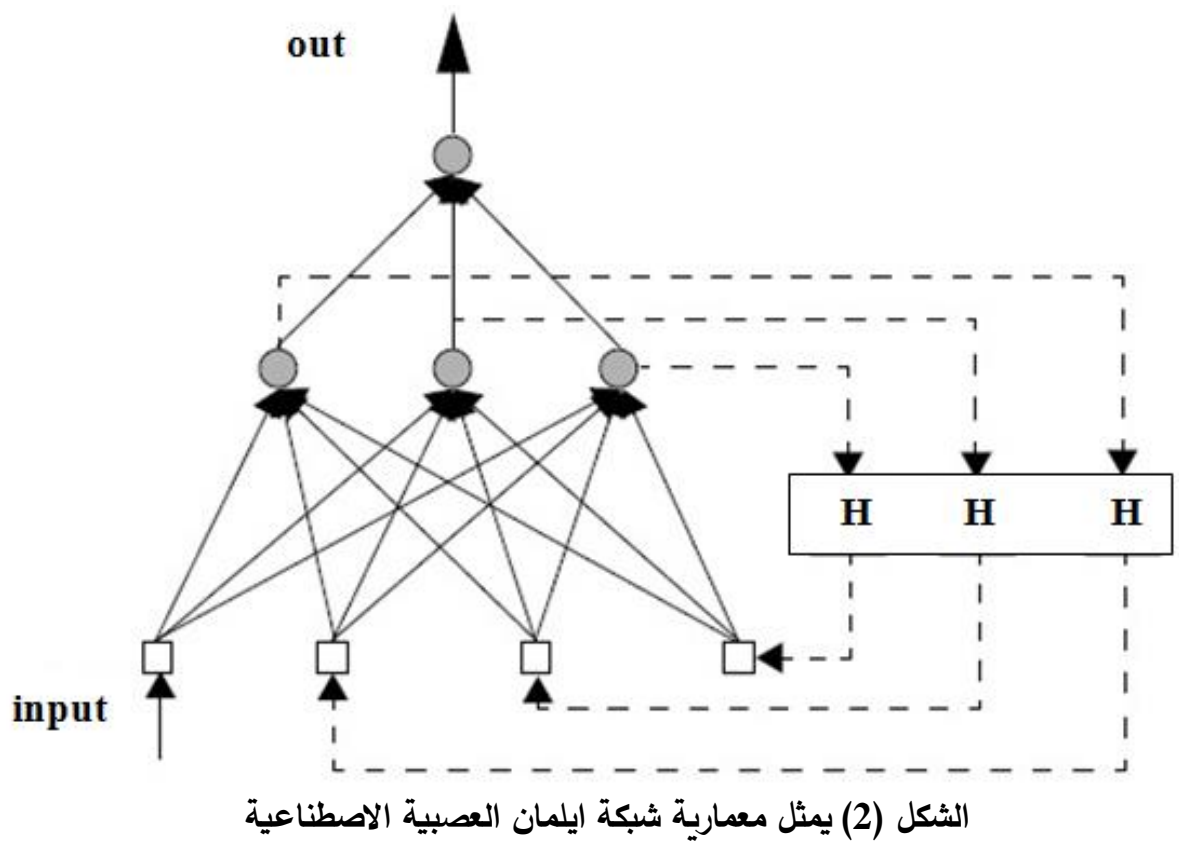

تتكون شبكة ايلمان العصبية من دالة التفعيل من النوع (tansig) في الطبقة المخفية ومن دالة التفعيل من النوع (purelin) في طبقة الإخراج, كما أن الطبقة المخفية تتطلب عدداً كافياً من وحدات المعالجة أو العقد يتناسب مع تعقيد المسألة المراد معالجتها, وقد تم استخدام (50) وحدة معالجة في بحثنا هذا, كما تتميز شبكة ايلمان العصبية الاصطناعية عن بقية الشبكات الاعتيادية ذات الطبقتين كون أن الطبقة الأولى فيها تمتلك اتصالاً متكرراً (Recurrent Connection), كما أن التأخير في الاتصال يخزن القيم من الخطوة السابقة والتي يمكن أن تستخدم في الخطوة الحالية [10] [11].

\section{4. آلية المعالجة في شبكة ايلمان العصبية الاصطناعية :}

تتكون شبكة ايلمان العصبية الاصطناعية من طبقة الإدخال وطبقة الإخراج بالإضافة إلى الطبقة المخفية, وتتميز بوجود وحدات معالجة ملحقة تدعى (Context) يكون عددها مساوياً لعدد وحدات المعالجة في الطبقة المخفية وتعطى لها قيماً صفرية في بداية المعالجة كما تعطى الأوزان المقابلة لها قيماً واحدية ثم تعدل ألثاء أثناء

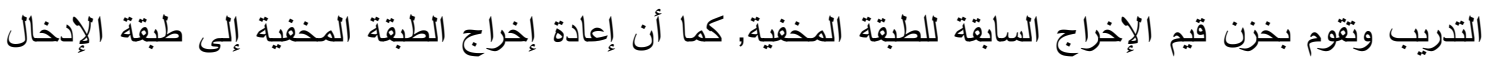

يعطي إمكانية بناء ذاكرة قصيرة الأمد تستعمل لتسريع عمل وتدريب الثبكة من خلال ضبط الأوزان [13] [1].

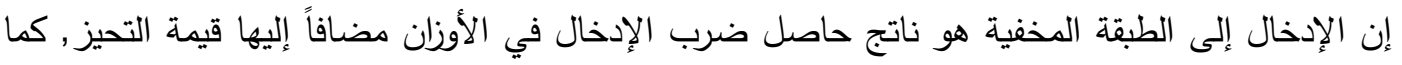
تعدل الأوزان عن طريق تكرار الانتثار الخلفي للخطأ لإعطاء الثبكة إمكانية معالجة الأنماط من خلال مراحل تكرارية مختلفة [4].

2. الأنموذج المقترح (ايلمان_ماركوف المخفي) (ELMAN_HM):

تم اقتراح خوارزمية مهجنة بين شبكة ايلمان (Elman) العصبية الاصطناعية وأنموذج ماركوف المخفي (HMM), وذلك من خلال إجراء تكامل في عملية المعالجة لبيانات الهثاشة والتي تتنمي إلى فضاء الأعداد الحقيقية ولايستطيع أنموذج ماركوف المخفي لوحده معالجتها كونه يتقبل إدخالات تنتمي إلى فضاء الأعداد الصحيحة الموجبة, حيث تم تهجين هذا الأنموذج باستخدام شبكة ايلمان العصبية الاصطناعية ذات القدرات 
الرياضية العالية في آلية معالجة البيانات, بالإضافة إلى كونها تتقبل البيانات التي تتمي إلى فضاءات الأعداد

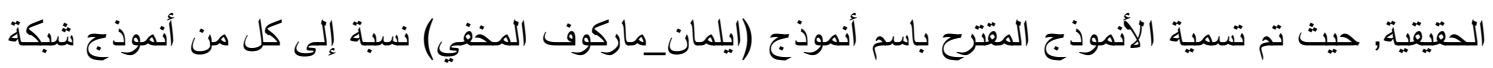
ايلمان وأنموذج ماركوف المخفي, حيث يتألف الأنموذج المقترح من مرحلتين أساسيتين من مراحل معالجة البيانات,

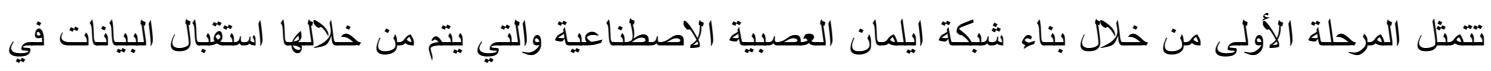

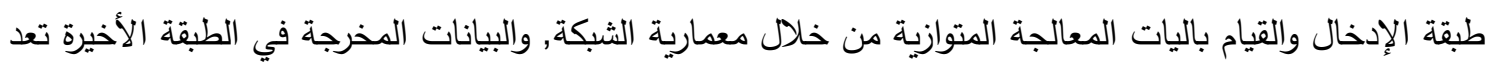
إدخالاً للمرحلة الثانية والتي تتم من خلال أنموذج ماركوف المخفي ويتم من خلالها تقدير معلمات الآلمات الأنموذج

ويصبح قادرا على تقبل البيانات الجديدة وتصنيفها.

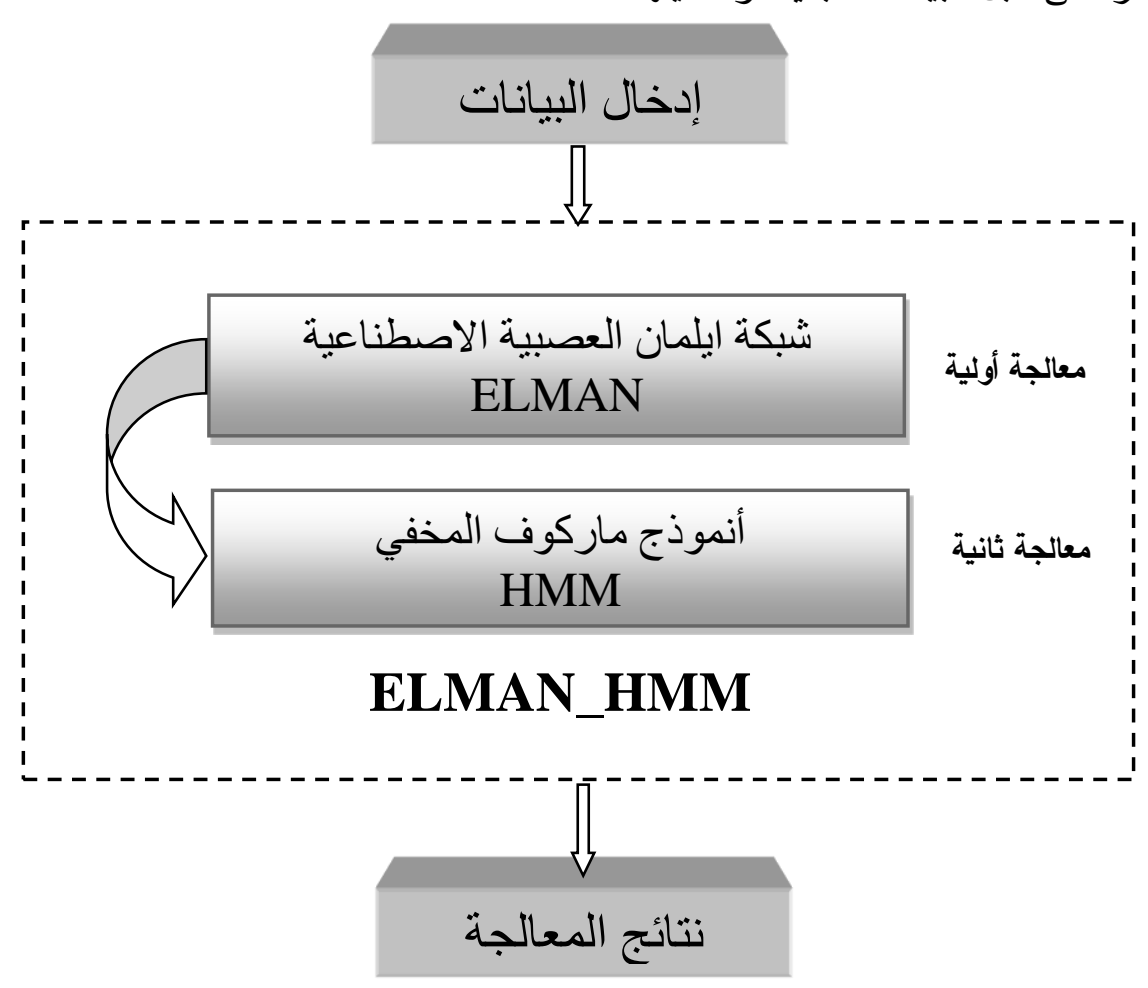

الثكل (3) مخطط عام يوضح مراحل المعالجة في الخوارزمية المقترحة (ELMAN_HMM).

1.5. تطبيق أنموذج ايلمان_ماركوف المخفي على بيانات هشاشة العظام

لقد تم استخدام وتطبيق أنموذج ايلمان_ماركوف المخفي على بيانات الهشاشة بوصفه إحدى النماذج الذكائية التي لاتعتمد على نوع محدد من بيانات الإدخال كما في أنموذج ماركوف المخفي الاعتيادي الذي يتطلب بيانات

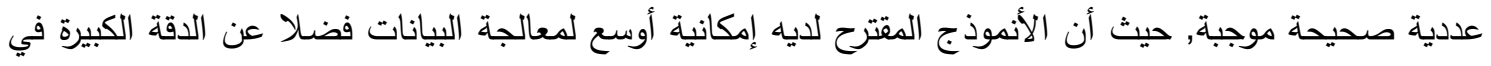

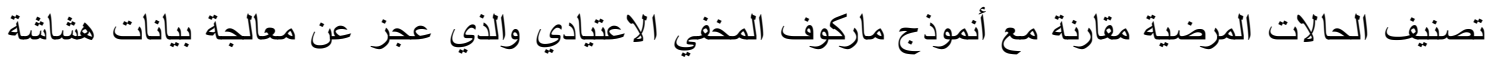
العظام كونها تحتوي على قيم تنتمي إلى فضاء الأعداد الحقيقية (Real Number) والتي لاتلائم فضاء الإدخال

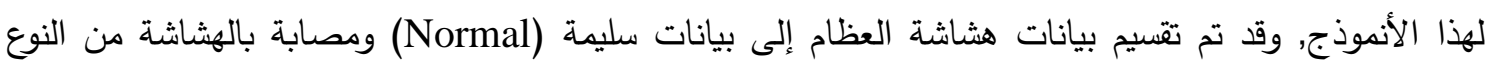
(Osteopenia)

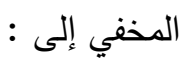


1. بيانات التدريب Training Data : حيث تتكون من (300) حالة منها (86) حالة من نوع (Normal) و

(116) حالة من نوع (Osteopenia) و (98) حالة من نوع (Osteoporosis). 2. بيانات الاختبار Testing Data : حيث تتكون من (44) حالة منها (14) حالة من (م) نوع (Normal) و

(15) حالة من نوع (Osteopenia) و (15) حالة من نوع (Osteoporosis).

إن الاختيار المناسب لمعلمات شبكة ايلمان العصبية الاصطناعية يعد من المسائل المهمة والمؤثرة في

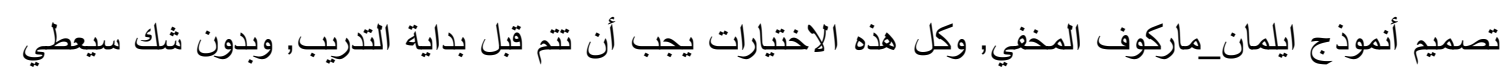

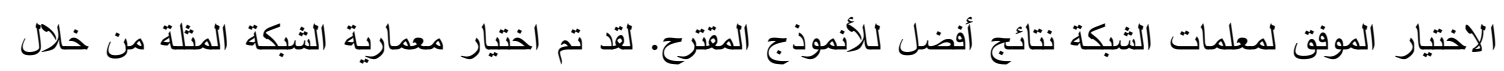
التجربة لحين إعطاء أفضل النتائج الممكنة بالنسبة لبيانات الإدخال. حيث تتكون معمارية هذه الثبكة من وحدات الإدخال والتي تمثل قراءات لحالات مرض هشاشة العظام المستخدمة في تدريب أنموذج الثبكة وعددها (17) قراءة

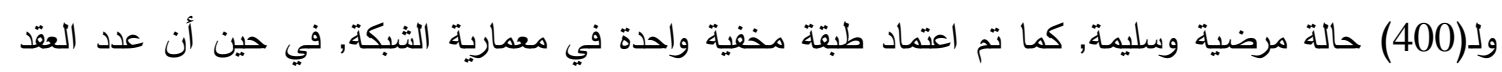
(Nodes) نوع (Hyperbolic Tangent Sigmoid), كما تم اعتماد الدالة الخطية (Linear Function) كدالة تتشيط في طبقة الإخراج.

3.الخوارزمية المقترحة في تصنيف بيانات هشاشة العظام:

إن الخطوات الرئيسية لتصنيف حالات أمراض هشاشة العظام باستخدام أنموذج ايلمان_ماركوف المخفي

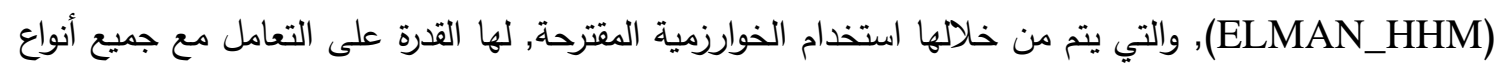
البيانات في فضاءات الأعداد, دون الاقتصار على البيانات العددية في فضاء الأعداد الصحيحة, وهذه ميزة تمتلكها

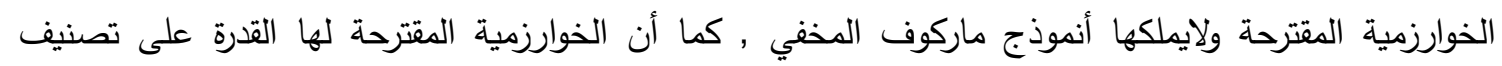

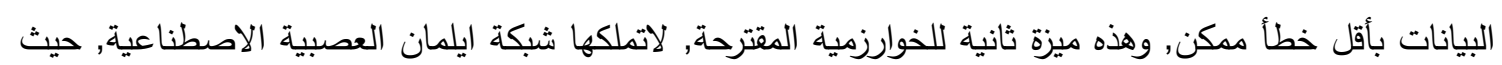
أن الخطوات الرئيسية للخوارزمية المقترحة تكون على النحو الآتي :

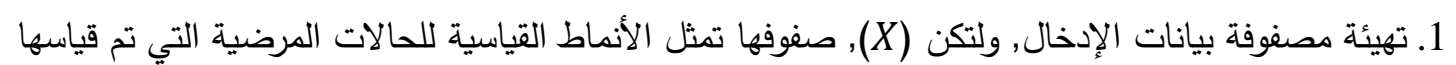

$$
\text { وأعمدتها تمثل الحالات المرضية المتنوعة. }
$$

2. تقسيم بيانات الإدخال إلى مجموعتين (X1) و وX2) حيث تمثل الأولى بيانات التدريب في حين تمثل الثانية

$$
\text { الحالات المستخدمة في اختبار الأنموذج. }
$$

3. إدخال بيانات التدريب على شبكة ايلمان العصبية الاصطناعية ومن ثم تدريب هذه البيانات من خلال

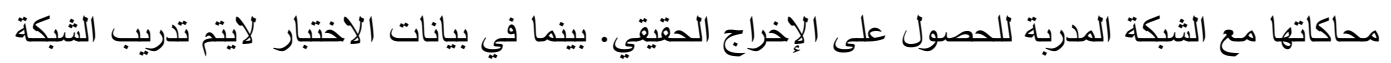

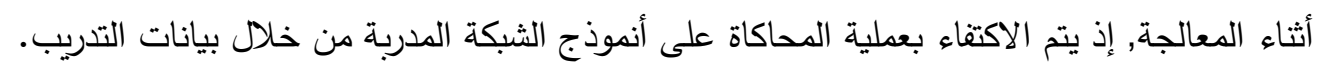

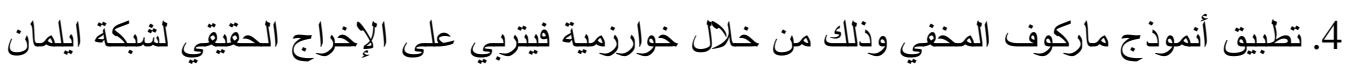
العصبية الاصطناعية. 5. مقارنة النتائج المستحصلة من الأنموذج المهن (ايلمان_ماركوف المخفي) في كل من بيانات التدريب والاختبار مع النتائج الحقيقية للحالات المرضية. 


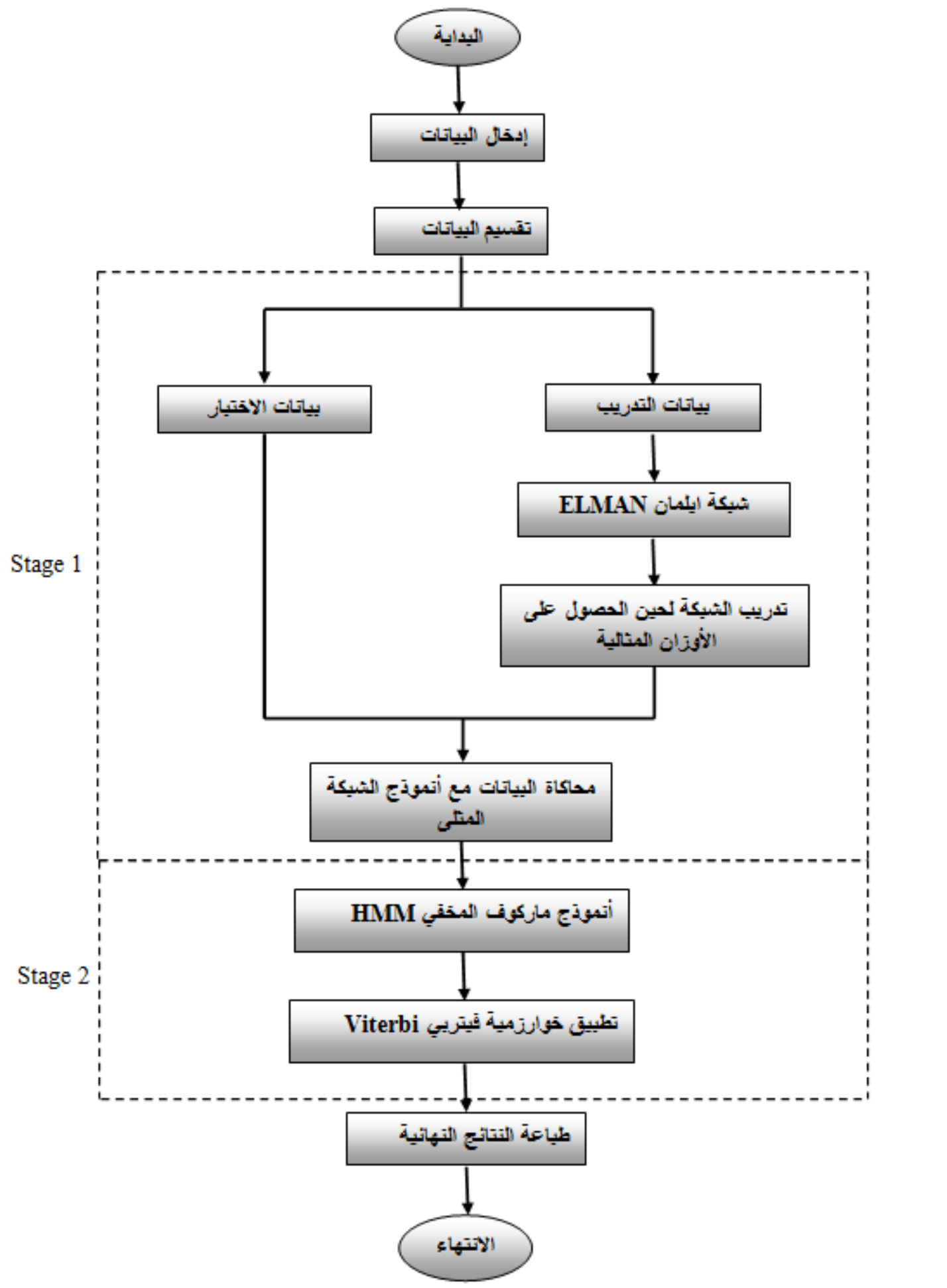

الثكل (4) مخطط انسيابي عام يوضح آلية عمل الخوارزمية المقترحة (ELMAN_HMM) 
4.مقارنة النتائج بين الخوارمية المهنة (ELMAN_HHM) والنماذج القياسية من خلال تصنيف أمراض هثاثة العظام

لقد تمت مقارنة الخوارزمية المقترحة (ELMAN_HHM) والتي يتم من خلالها تهجين شبكة ايلمان العصبية الاصطناعية و أنموذج ماركوف المخفي, مع كل من شبكة ايلمان العصبية الاصطناعية القياسية من فن من فئن

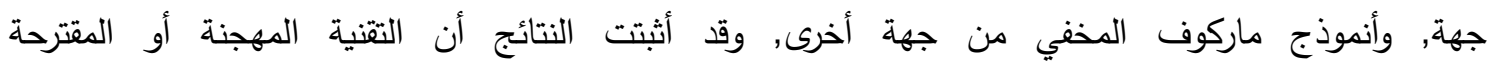
هي الأفضل من ناحية دقة النتائج المطلوبة التي يستدل عليها من خلال مقياس معدل مربع الخطأ (MSE) بالنسبة لبيانات الاختبار والتدريب. والجدول (1) يوضح نتائج هذه الدقارنة : الجدول (1) مقارنة بين التقنية المهجنة (ELMAN_HHM) مع كل من شبكة ايلمان العصبية وأنموذج ماركوف المخفي

\begin{tabular}{|c|c|c|c|}
\hline $\begin{array}{c}\text { معدل مربع الخطأ لبيانات الاختبار } \\
\text { (MSE_Testing) }\end{array}$ & $\begin{array}{c}\text { معدل مربع الخطأ لبيانات } \\
\text { (MSE_Training) } \\
\text { (MSE_Trating }\end{array}$ & $\begin{array}{l}\text { تقبل فضاء الإدخال } \\
\text { (Input Space) }\end{array}$ & نوع التقنية الذكائية المستخدمة \\
\hline 0.0682 & 0.05 & نعم & $\begin{array}{c}\text { التقنية المهجنة } \\
\text { (ELMAN_HHM) }\end{array}$ \\
\hline 0.2307 & 0.2107 & نعم & شبكة ايلمان العصبية \\
\hline ----------- & ------ & لايمكن & $\begin{array}{c}\text { أنموذج ماركوف المخفي } \\
\text { (HHM) }\end{array}$ \\
\hline
\end{tabular}

إن نتائج التهجين المبينة في الجدول (1) والمتمثلة بتقنية ELMAN_HHM) أثبتت أنها هي الأفضل من خلال مقياس (MSE), من شبكة ايلمان العصبية الاصطناعي ومن خلال بيانات الاختبار والتدريب فضلاً

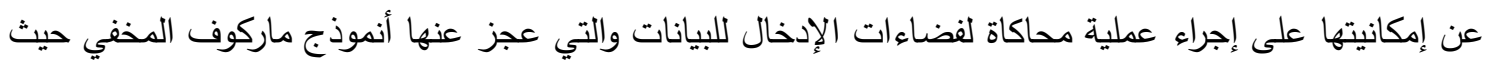
أن هذا الأنموذج لايستطيع معالجة بيانات الهثاشة كونها تتنمي إلى فضاء الأعداد الحقيقية, لذا لايمكن استخدامه

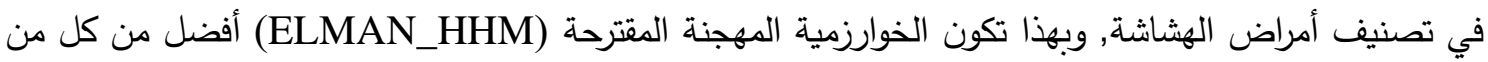
تقنيتي شبكة ايلمان العصبية وأنموذج ماركوف المخفي. كما يبين الثكل (4) مقارنة لعدد الحالات المصنفة بشكل صحيح في كل من تقنيتي شبكة ايلمان العصبية (ELMAN) والتتنية المهجنة (ELMAN_HMM) بالنسبة

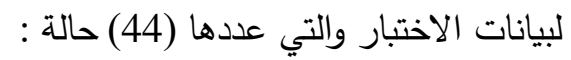


مقارنة بين تقنيتي (ELMAN) و (ELMAN_HMM) في تصنيف بيانات هثاثة العظام

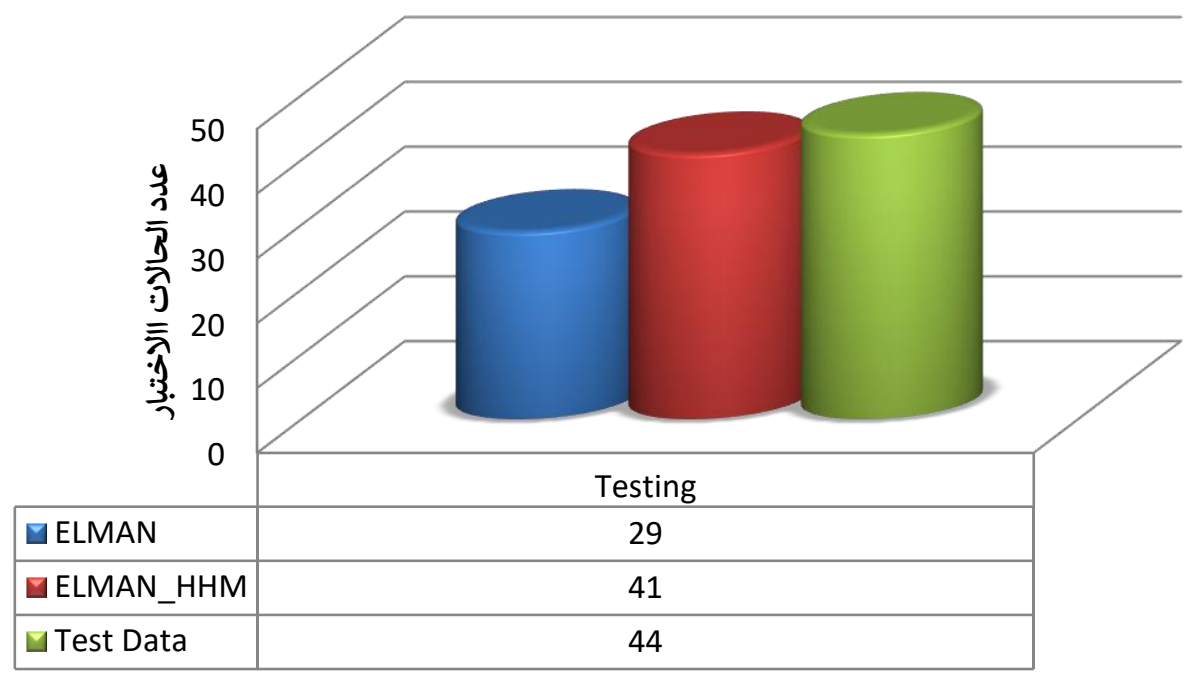

الثكل (4) يبين مقارنة بين عدد حالات الاختبار المصنفة بثكل صحيح في كل من تقنية (ELMAN) والتقنية المهجنة (ELMAN_HMM)

5. الاستنتاجات والتوصيات

$$
\text { من خلال النتائج في الجدول (1) نستتتج مائتي : }
$$

1. لقد اثبت الأنموذج المهجن المقترح (ELMAN_HMM) في الجدول (1) إمكانية عالية في تصنيف بيانات الهثاشة وذلك من خلال مقياس (MSE) مقارنة مع شبكة ايلمان العصبية الاصطناعية وفي كل من بيانات

$$
\text { التدريب والاختبار • }
$$

2. إمكانية الأنموذج المقترح (ELMAN_HMM) التعامل مع جميع أنواع المدخلات في فضاء الإدخال

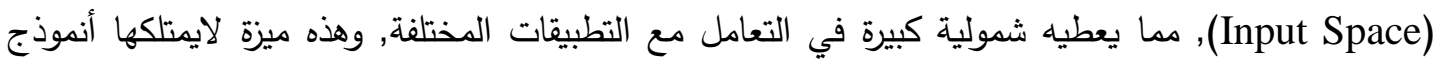

$$
\text { ماركوف المخفي القياسي. }
$$

3. أثثت شبكة ايلمان العصبية الاصطناعية أنها أكثر عمومية من أنموذج ماركوف المخفي في التعامل مع التطبيقات كونها تتقبل جميع أنواع الفضاءات التي تتنمي إليها البيانات.

$$
\text { كما نوصي بالآتي: }
$$

1. نوصي باستخدام تهجين بين أنموذج ماركوف المخفي وشبكات عصبية أخرى لها إمكانيات في معالجة

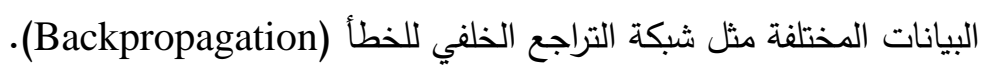
2. نوصي بتوظيف الطريقة المقترحة كنظام مساعد للطبيب في المراكز الطبية المختلفة واستخدامها على بيانات

$$
\text { تتنمي إلى فضاءات متعددة. }
$$




$$
\begin{aligned}
& \text { المصادر } \\
& \text { جبريني, مصون نبهان حمصي ،(2010)، "نظام تفاعلي ذكي من اجل التعليم على } \\
& \text { الثبكة العنكبوتية", أطروحة دكتوراه غير منشورة، كلية العلوم، جامعة حلب، سوريا. } \\
& \text { الخياط, باسل يونس،(2010)، "النمذجة الماركوفية مع تطبيقات عملية", الجزء الثاني, }
\end{aligned}
$$

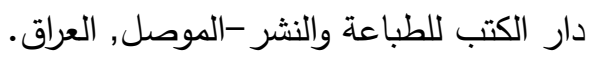

$$
\begin{aligned}
& \text { زمزوم, فاطمة محمود حسن(2010)، "استخدام النمذجة الماركوفية في المعلوماتية } \\
& \text { الحياتية", رسالة ماجستير غير منشورة، ، كلية علوم الحاسوب والرياضيات, جامعة } \\
& \text { الموصل، العراق. } \\
& \text { عيسى، علام زكي ،(2000)، "الثبكات العصبية، البنية الهندسية-الخوارزميات- } \\
& \text { التطبيقات"، الطبعة الأولى، شعاع للنشر والعلوم،. حلب، سوريا. } \\
& \text { محمد, سوزان حسن ،(2011)، " تأثير استخدام مرشحات كثف الحواف على أداء }
\end{aligned}
$$

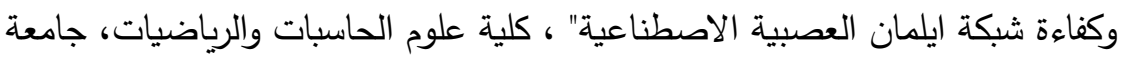

$$
\begin{aligned}
& \text { الموصل ، العراق. }
\end{aligned}
$$

[6] Batzoglou,S.، (2010)، "Hidden Markov Models and the Viterbi algorithem" Scribed by John C.Mu .

[7] Fonzo, V. , Aluffi-Pentini, F., and Parisi, V، (2007) ‘" Hidden Markov Models in Bioinformatics", Vol. 2, No. 1, Euro. Bio. Park, University di Roma, Roma, Italy.

[8] http://en.wikipedia.org/wiki/Osteoporosis.

[9] Medsker L.R. \& Jain L.C.,(2001)," RECURRENT NEURAL NETWORKS: Design and Applications", CRC Press Boca Raton London New York Washington, D.C.

[10] Ramadevi R., Sheela R. B. \& Prakash V. ,(2012), "Role of Hidden Neurons in an Elman Recurrent Neural Network in Classification of Cavitation Signals", International Journal of Computer Applications (0975 - 8887), Volume 37- No.7.

[11] Ramo R.M. \& Ramo F.M. ,(2011),"Face Detection Using Neural Networks", The Fourth Scientific Conference of the College of Computer Science \& Mathematics pp [336-350].

[12] Wolfram U., Schwen L.O. \& Simon U. ,(2009) ," Statistical Osteoporosis Models Using Composite Finite Elements: A Parameter Study",Submitted to Journal of Biomechanics.

[13] Zeng Z., (2012),"Multi-step Predictions Based on TD-DBP ELMAN Neural Network for Wave Compensating Platform", Advances in CSIE, Vol. 2, AISC 169, pp. 431-436. @ Springer-Verlag Berlin Heidelberg. 
ملحقى (1) يعثل بياتات الاختبار الهرض هثائدة العظام المستقدهة في البحث

\begin{tabular}{|c|c|c|c|c|c|c|c|c|c|c|c|c|c|c|c|c|c|}
\hline y & $\mathrm{Xl}$ & $\mathrm{x} 2$ & $\mathrm{x} 3$ & $\mathrm{X} 4$ & $\mathrm{X} 5$ & $\mathrm{x} 6$ & $\times 7$ & $\mathrm{X} 8$ & $\mathrm{x} 9$ & $\mathrm{x} 10$ & X11 & $\mathrm{X} 12$ & \begin{tabular}{|l|}
$\mathrm{X} 13$ \\
\end{tabular} & \begin{tabular}{|l|}
$\mathrm{X} 14$ \\
\end{tabular} & \begin{tabular}{|l|}
$\mathrm{X} 15$ \\
\end{tabular} & $\mathrm{x} 16$ & $\mathrm{x} 17$ \\
\hline 3 & 1 & 1 & 1 & 1 & 1 & 0 & 0 & 0.5 & 0.333333 & 0 & 1 & 0.344828 & 1 & 0.2 & 0 & 1 & 0.75 \\
\hline 3 & 1 & 1 & 1 & 1 & & 1 & 1 & 0 & 0.333333 & 0 & 1 & 0.275862 & 1 & 0.4 & 0 & 1 & 0.75 \\
\hline 3 & 1 & 0.5 & 1 & 1 & & 0 & 0 & 0.5 & 0.666667 & 0 & 1 & 0.344828 & 1 & 0 & 0 & 1 & 0.75 \\
\hline 3 & 1 & 0 & 1 & 1 & & 1 & 0 & 0.5 & 0.666667 & 0 & 0.5 & 0.275862 & 4 & 0 & 0 & 1 & 0.5 \\
\hline 3 & 1 & 0 & 1 & 1 & & 1 & 1 & 0.5 & 0.666667 & 0 & 1 & 0.206897 & & 0 & 0 & 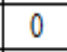 & 0.75 \\
\hline 3 & 1 & 1 & 1 & 1 & & 1 & 0 & 0. & 333333 & 1 & & 586207 & & 1 & 0 & 1 & 0.75 \\
\hline 3 & 1 & 0 & 1 & 1 & & 0 & 1 & 0 & 33333 & 0 & 1 & 0.275862 & & & 0 & 1 & 1 \\
\hline 3 & 1 & 0 & 1 & 1 & & 1 & 1 & 0.5 & 1 & 0 & 4 & 0.37931 & & 1 & 1 & 4 & 0.5 \\
\hline 3 & 1 & 1 & 1 & 1 & & 0 & 1 & 0 & 0.666667 & 0 & 1 & 0.103448 & 1 & 0.4 & 1 & 1 & 0.75 \\
\hline 3 & 1 & 1 & 1 & 1 & & 1 & 0 & 1 & 0.333333 & 1 & 1 & 0.241379 & 0 & 1 & 1 & 1 & 0.5 \\
\hline 3 & 1 & 0 & 1 & 1 & & 0 & 1 & 0 & 0.666667 & 0 & 1 & 0.275862 & 1 & 0.4 & 1 & 1 & 0.5 \\
\hline 3 & 1 & 0.5 & 1 & 1 & & 1 & 1 & 0.5 & 0.666667 & 1 & 1 & 0.413793 & 1 & 0 & 1 & 1 & 0.75 \\
\hline 3 & 1 & 1 & 1 & 1 & & 1 & 0 & 0.5 & 0.666667 & 0 & 1 & 0.448276 & 1 & 0.4 & 1 & 1 & 0.75 \\
\hline 3 & 1 & 0 & 1 & 1 & & 0 & 0 & 0.5 & 0.666667 & 0 & 0.5 & 0.241379 & 1 & & 1 & 1 & 0.75 \\
\hline 3 & 1 & 0 & 1 & 1 & & 1 & 1 & 0 & 666667 & 0 & \begin{tabular}{|l|}
1 \\
\end{tabular} & 0.241379 & 1 & & 1 & 1 & 0.5 \\
\hline 1 & 0 & 1 & 1 & 1 & & 1 & 1 & 1 & 7 & 0 & & 0.206897 & & & 1 & & 0 \\
\hline 1 & 0 & 1 & 1 & 1 & & 1 & 0 & 0 & 333333 & 0 & 1 & 0.517241 & & 0 & 1 & 1 & 0.75 \\
\hline 1 & 0 & 1 & 1 & 1 & & 1 & 1 & 0 & 0.666667 & 0 & 1 & 0.448276 & 1 & 0 & 1 & 0 & 0.25 \\
\hline 1 & 0 & 1 & 0 & 1 & & 1 & 1 & 1 & 0.333333 & 0 & 1 & 0.689655 & 0 & 1 & 1 & 1 & 0.25 \\
\hline 1 & 0 & 0 & 1 & 1 & & 1 & 1 & 0.5 & 0.666667 & 0 & 1 & 0.275862 & 1 & 0 & 1 & 1 & 0.5 \\
\hline 1 & 0 & 1 & 1 & 1 & & 1 & 0 & & 6667 & 0 & 1 & & & & 1 & & 0.5 \\
\hline 1 & 0 & 0 & 1 & 1 & & 1 & 1 & 0 & 33333 & 0 & 1 & 0.448 & & & 1 & & 0.5 \\
\hline 1 & 0 & 1 & 1 & 1 & & 1 & 1 & 0 & 0.333333 & 0 & 1 & 0.310345 & & 0.4 & 1 & & 0.5 \\
\hline 1 & 0 & 1 & 1 & 1 & & 1 & 1 & 1 & 0.333333 & 0 & 1 & 0.344828 & 1 & 0.8 & 1 & 1 & 0.25 \\
\hline 1 & 0 & 1 & 1 & 1 & & 1 & 1 & 0 & 0.666667 & 1 & 1 & 0.655172 & 1 & 0 & 1 & 1 & 0.75 \\
\hline 1 & 0 & 1 & 0.5 & 1 & & 1 & 1 & 1 & 0.333333 & 0 & 1 & 0.37931 & 0 & 1 & 1 & 1 & 0.25 \\
\hline 1 & 0 & 1 & 1 & 1 & 1 & 1 & 1 & 1 & 1 & 0 & 1 & 0.275862 & 0 & 1 & 1 & 1 & 0.5 \\
\hline 1 & 0 & 1 & 1 & 1 & 1 & 1 & 1 & 0 & 1 & 0 & 1 & 0.689655 & 1 & 0 & 0.5 & 0 & 0.75 \\
\hline 1 & 0 & 1 & 1 & 1 & & 1 & 0.5 & 1 & .666667 & 1 & 1 & 0.517 & 1 & 0.4 & 1 & 1 & 0.25 \\
\hline 2 & 0 & 1 & 1 & 1 & & 1 & 1 & 0 & & 0 & 1 & 14 & 0 & & 1 & 1 & 0.5 \\
\hline 2 & 0 & 1 & 1 & 1 & & 1 & 0 & 0.5 & 666667 & 0 & 1 & 21 & 1 & 0.4 & 1 & 1 & 0.75 \\
\hline 2 & 0 & 1 & 1 & 1 & 1 & 1 & 0 & 1 & 0 & 0 & 1 & 0.206897 & 1 & 0 & 1 & 1 & 0 \\
\hline 2 & 0 & 1 & 1 & 1 & 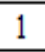 & 1 & 1 & 0 & 333333 & 0 & 1 & 0.758621 & 1 & 1 & 1 & 1 & 0.75 \\
\hline 2 & 0 & 1 & 1 & 1 & & 1 & 1 & 0 & 0.333333 & 0 & 1 & 0.310345 & 0 & 1 & 1 & 1 & 0.25 \\
\hline 2 & 0 & 1 & 1 & 1 & 1 & 1 & 1 & 0.5 & 0.666667 & 0 & 1 & 0.37931 & 1 & 0 & 1 & 1 & 0.5 \\
\hline 2 & 0 & 1 & 1 & 1 & 1 & 1 & 1 & 0 & 0.333333 & 0 & 1 & 0.275862 & 1 & 0.6 & 1 & 1 & 0.75 \\
\hline 2 & 0 & 1 & 1 & 1 & 1 & 1 & 0 & 0.5 & 0.666667 & 0 & 1 & 0.310345 & 1 & 0 & 1 & 1 & 0.5 \\
\hline 2 & 0 & 1 & 1 & 1 & 1 & 1 & 1 & 0.5 & .666667 & 0 & 1 & 0.344828 & 1 & 0.2 & 1 & 1 & 1 \\
\hline 2 & 0 & 0 & 1 & 1 & & 0 & 1 & 0 & 666667 & 0 & 1 & 0.48 & 1 & 0 & 1 & 1 & 0.75 \\
\hline 2 & 0 & 1 & 1 & 1 & 1 & 1 & 1 & 0.5 & 1 & 1 & 1 & 0.413793 & 1 & 0.2 & 1 & 1 & 0.5 \\
\hline 2 & 0 & 1 & 1 & 1 & & 1 & 0 & 0 & .666667 & 0 & 1 & 0.275862 & 1 & 0.4 & 1 & 1 & 0.25 \\
\hline 2 & 0 & 1 & 1 & 1 & & 1 & 1 & 0 & 0.666667 & 1 & 1 & 0.896552 & 1 & 0 & 1 & 1 & 0.5 \\
\hline 2 & 0 & 1 & 1 & 1 & & 1 & 1 & 0.5 & 0.666667 & 0 & 1 & 0.448276 & 1 & 0 & 1 & 0 & 1 \\
\hline 2 & 0 & 1 & 1 & 1 & 1 & 1 & 1 & 1 & 0.666667 & 0 & 1 & 0.344828 & 1 & 0.4 & 1 & 0 & 0.25 \\
\hline
\end{tabular}


ملحتى (2) يمثل بياتات التثريب أهرض هثائدة المثام المستقدهة في البحث

\begin{tabular}{|c|c|c|c|c|c|c|c|c|c|c|c|c|c|c|c|c|c|}
\hline & $\mathrm{xl}$ & $\mathrm{x} 2$ & $\mathrm{x} 3$ & $\mathrm{X} 4$ & $\mathrm{X} 5$ & $\mathrm{X} 6$ & $\mathrm{x} 7$ & $\mathrm{x} 8$ & $\mathrm{X} 9$ & $\mathrm{x} 10$ & Xl1 & $\mathrm{x} 12$ & \begin{tabular}{|l|l|}
$\mathrm{x} 13$ \\
\end{tabular} & \begin{tabular}{|l|l|}
$\mathrm{X} 14$ \\
\end{tabular} & \begin{tabular}{|l|}
$\mathrm{X} 15$ \\
\end{tabular} & $\mathrm{X} 16$ & $\mathrm{x} 17$ \\
\hline 3 & 1 & 0 & 1 & 1 & 1 & 1 & 1 & \begin{tabular}{|l|}
0.5 \\
\end{tabular} & 0.333333 & 1 & 1 & 0.551724 & 1 & $\begin{array}{l}0 \\
\end{array}$ & 1 & 1 & 1 \\
\hline 3 & 1 & 0 & 1 & 1 & 1 & 0 & 1 & 0.5 & 0.666667 & 0 & 1 & 0.103448 & 1 & \begin{tabular}{|l|} 
\\
\end{tabular} & 1 & 1 & 0.75 \\
\hline 3 & 1 & 0 & 1 & 1 & 1 & 0 & 1 & 0 & 0.666667 & 0 & 1 & 0.137931 & 1 & 0 & 1 & 1 & 0.75 \\
\hline 3 & 1 & 0 & 0 & 1 & 1 & 1 & 1 & 0.5 & 0.666667 & 1 & 1 & 0.275862 & 1 & 0 & 1 & 1 & 0.75 \\
\hline 3 & 1 & 1 & 1 & 0 & 1 & 1 & 1 & 0 & 0.6666667 & 1 & 1 & 0.344828 & 1 & 0 & 1 & 1 & 1 \\
\hline 3 & 1 & 0.5 & 1 & 0 & 1 & 1 & 1 & 0 & 0.666667 & 0 & \begin{tabular}{|l|}
0.5 \\
\end{tabular} & 0.241379 & 1 & 0 & 1 & 1 & 0.75 \\
\hline 3 & 1 & 1 & 1 & 1 & 1 & 1 & 1 & 0 & 0.666667 & 0 & 1 & 0.448276 & 1 & 0.2 & 1 & 1 & 0.25 \\
\hline 3 & 1 & 1 & 1 & 1 & 0 & 0 & 1 & 0 & 1 & 1 & 1 & 0.068966 & 0 & 1 & 0.5 & 1 & 0.5 \\
\hline 3 & 1 & 0.5 & 1 & 1 & 1 & 0 & 1 & 0 & 0.666667 & 0 & 1 & 0.344828 & 1 & 0.4 & 1 & 1 & 0.5 \\
\hline 3 & 1 & 1 & 0 & 1 & 1 & 1 & 1 & 0 & 0 & 0 & 1 & 0.241379 & 0 & 1 & 1 & 1 & 0.25 \\
\hline 3 & 1 & 1 & 1 & 1 & 1 & 1 & 1 & 0 & 0.666667 & 0 & 0.5 & 0.448276 & 1 & 0 & 1 & 1 & 0.25 \\
\hline 3 & 1 & 0 & 1 & 1 & 1 & 0 & 1 & 0 & 0 & 0 & 1 & 0.275862 & 1 & \begin{tabular}{|l|} 
\\
\end{tabular} & 1 & 1 & 0.5 \\
\hline 3 & 1 & 1 & 1 & 0 & 1 & 0 & 1 & 0 & 0.666667 & 0 & 1 & 0 & 1 & 0 & 1 & 1 & 0.5 \\
\hline 3 & 1 & 0.5 & 1 & 1 & 1 & 0 & 0 & 0 & 0.333333 & 0 & 1 & 0.310345 & 0 & 1 & 1 & 1 & 0.75 \\
\hline 3 & 1 & 0 & 1 & 0 & 1 & 1 & 1 & 0 & 0.333333 & 0 & 1 & 0.448276 & 1 & 1 & 1 & 1 & 0.75 \\
\hline 3 & 1 & 0 & 1 & 1 & 1 & 1 & 1 & 0 & 0.666667 & 0 & 1 & 0.448276 & 1 & 0 & 1 & 1 & 0.75 \\
\hline 3 & 1 & 1 & 1 & 1 & 1 & 1 & 1 & 0 & 0.666667 & 1 & 1 & 0.241379 & 1 & 0 & 1 & 1 & 0.75 \\
\hline 3 & 1 & 1 & 1 & 1 & 1 & 1 & 1 & 0 & 0.666667 & 1 & 1 & 0.517241 & 1 & 0 & 1 & 1 & 0.5 \\
\hline 3 & 1 & 1 & 1 & 1 & 1 & 1 & & & 0.666667 & 0 & 0.5 & 0.517241 & 1 & 0 & & 1 & 0.75 \\
\hline 3 & 1 & 0 & 1 & 1 & 1 & 1 & 1 & 0 & 0.666667 & 0 & 1 & 0.482759 & 1 & 0 & 1 & 1 & 0.5 \\
\hline 3 & 1 & 0 & 1 & 1 & 1 & 1 & 1 & 0 & 0.666667 & 1 & 1 & 0.517241 & 1 & 0 & 1 & 1 & 0.75 \\
\hline 3 & 1 & 1 & 1 & 1 & 1 & 0 & 1 & 0 & 0 & 1 & 1 & 0.275862 & 0 & 1 & 1 & 1 & 0.5 \\
\hline 3 & 1 & 1 & 1 & 1 & 1 & 1 & 1 & 0.5 & 0.666667 & 0 & 1 & 0.482759 & 1 & 0.2 & & 1 & 0.5 \\
\hline 3 & 1 & 1 & 1 & 1 & 1 & 0 & 1 & & 0.333333 & 0 & 1 & 0.551724 & 1 & 1 & & 1 & 0.75 \\
\hline 3 & 1 & 0.5 & 1 & 0 & 1 & 0 & 1 & 0.5 & 0.666667 & 0 & 1 & 0.034483 & 1 & 0 & 1 & 1 & 1 \\
\hline 3 & 1 & 0 & 1 & 1 & 1 & 0 & 1 & 0 & 1 & 1 & 1 & 0.172414 & 0 & 1 & 0.5 & 1 & 0.5 \\
\hline 3 & 1 & 0.5 & 0 & 1 & 1 & 1 & 1 & 0.5 & 0.666667 & 1 & 1 & 0.448276 & 1 & 0 & 1 & 1 & 1 \\
\hline 3 & 1 & 1 & 1 & 1 & 1 & 1 & 1 & 0 & 0.666667 & 0 & \begin{tabular}{|l|}
0.5 \\
\end{tabular} & 0.448276 & 1 & 0 & 1 & 0 & 0.5 \\
\hline 3 & 1 & 1 & 0 & 1 & 1 & 0 & 1 & & 0.333333 & 0 & 1 & 0.241379 & 1 & 0.4 & 1 & 1 & 0.75 \\
\hline 3 & 1 & 1 & 1 & 1 & 1 & 1 & 1 & 0.5 & 0.666667 & 0 & 1 & 0.344828 & 1 & 0 & 1 & 1 & 0.5 \\
\hline 3 & 1 & 1 & 1 & 1 & 1 & 1 & 1 & 0. & 0.666667 & 0 & 1 & 0.413793 & 1 & 0 & 1 & 1 & 0.5 \\
\hline 3 & 1 & 1 & 1 & 1 & 1 & 0 & 1 & 0.5 & 0.666667 & 1 & 1 & 0.482759 & 1 & 0 & 1 & 1 & 0.75 \\
\hline 3 & 1 & 1 & 1 & 1 & 1 & 1 & 1 & 0 & 1 & 0 & \begin{tabular}{|l|}
0.5 \\
\end{tabular} & 0.37931 & 1 & \begin{tabular}{|l|}
0.4 \\
\end{tabular} & & 1 & 0.5 \\
\hline 3 & 1 & 1 & 1 & 1 & 1 & 1 & 0 & 0.5 & 0.666667 & 1 & 1 & 0.517241 & 0 & 1 & 1 & 1 & 0.75 \\
\hline 3 & 1 & 1 & 0 & 0 & 1 & 0 & 1 & 0.5 & 0.666667 & 1 & 1 & 0.241379 & 1 & 0 & 1 & 1 & 1 \\
\hline 3 & 1 & 0 & 1 & 1 & 1 & 1 & 1 & 0 & 0.666667 & 0 & 1 & 0.241379 & 1 & 0 & 1 & 1 & 0.75 \\
\hline 3 & 1 & 1 & 1 & 1 & 1 & 1 & 0 & 0.5 & 0.666667 & 0 & 1 & 0.172414 & 1 & 0 & 1 & 1 & 0.75 \\
\hline 3 & 1 & 1 & 1 & 1 & 1 & 1 & 1 & 0 & 0.666667 & 1 & \begin{tabular}{|l|}
0.5 \\
\end{tabular} & 0.172414 & 1 & 0 & 1 & 1 & 0.75 \\
\hline 3 & 1 & 1 & 1 & 1 & 1 & 1 & 0 & 0.5 & 0.666667 & 0 & 1 & 0.517241 & 1 & 0 & 1 & 1 & 0.75 \\
\hline 3 & 1 & 1 & 1 & 1 & 1 & 1 & 1 & 0 & 0.666667 & 1 & 1 & 0.344828 & 1 & 0 & 1 & 0 & 0.75 \\
\hline 3 & 1 & 0 & 1 & 1 & 1 & 1 & 0 & 0.5 & 0.666667 & 1 & 1 & 0.310345 & 1 & 0 & 1 & 1 & 0.5 \\
\hline 3 & 1 & 1 & 1 & 1 & 1 & 1 & 1 & 0.5 & 0.666667 & 0 & 1 & 0.344828 & 1 & 0 & 1 & 0 & 0.5 \\
\hline 3 & 1 & 0 & 1 & 1 & 1 & 1 & 1 & 0.5 & 0.666667 & 0 & 1 & 0.37931 & 1 & 0 & 1 & 1 & 0.75 \\
\hline
\end{tabular}




\begin{tabular}{|c|c|c|c|c|c|c|c|c|c|c|c|c|c|c|c|c|c|}
\hline & & & & & & & 1 & & 666667 & & 1 & 0.137931 & 1 & 0.4 & 1 & & \\
\hline 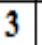 & 1 & 0.5 & 1 & 1 & & 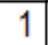 & 1 & 0.5 & 666667 & & 0 & 0.241379 & 1 & 0 & 1 & 0 & 0.75 \\
\hline & 1 & 1 & 0 & 1 & & 0 & 0.5 & 0 & 0 & & 1 & 0.344828 & 1 & 0.4 & 1 & & 0.5 \\
\hline & 1 & & & & & & & & 666667 & & 1 & 0.103448 & 1 & & & & 0.75 \\
\hline & 1 & & & & & & & 0.5 & 6667 & & & & 1 & & & & .75 \\
\hline 3 & 1 & 1 & 1 & 1 & & & 1 & & 33333 & & 1 & 0.172414 & 1 & & 1 & 1 & 0.25 \\
\hline 3 & 1 & 1 & & 1 & 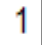 & 1 & 1 & 0.5 & 1 & & 1 & 0.310345 & 1 & 1 & 0.5 & & \\
\hline 3 & 1 & & & 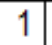 & & & ) & 0.5 & 333333 & & 1 & 0.586207 & 1 & 0.4 & 1 & & 0.75 \\
\hline 3 & 1 & & . & 1 & & & & & 66667 & & 0.5 & 0.344828 & 1 & & 1 & & \\
\hline 3 & 1 & 0 & 1 & 1 & 1 & & & & 1 & & 1 & 0.310345 & 1 & 0 & 0.5 & & 0.5 \\
\hline 3 & 1 & 0 & 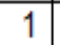 & ( & & & & 0 & 333333 & & 1 & 0.37931 & 1 & 0 & & & 0.5 \\
\hline 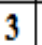 & 1 & 1 & 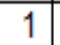 & 1 & 7 & 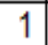 & & 0 & 0.666667 & & 1 & 0.413793 & 1 & 0.6 & 1 & & 0.5 \\
\hline 3 & 1 & 1 & 1 & 1 & 1 & 1 & & & 666667 & & 1 & 0.41 & 1 & & 1 & & 0.75 \\
\hline 3 & 1 & & 1 & 0 & & & 4 & 0.5 & 0 & & 1 & 0.31 & 1 & 0.6 & 0.5 & & 0.5 \\
\hline 3 & 1 & 1 & 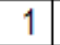 & 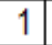 & & & 1 & & 666667 & & 1 & 0.310 & 1 & 0 & & & 0.5 \\
\hline 3 & 1 & 1 & & 1 & 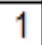 & 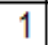 & & & 0.333333 & & 1 & 0.482759 & 1 & 0 & 1 & 1 & 0.75 \\
\hline 3 & 1 & 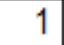 & 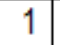 & 1 & & & & & 666667 & & & 0.37931 & 1 & 0.4 & & & \\
\hline 3 & 1 & -1 & & 1 & & & & 0 & 666667 & & 1 & 0.448276 & 1 & 0.2 & & & 0.5 \\
\hline 3 & 1 & 1 & 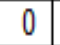 & 1 & & & & & .666667 & & 1 & 0.758621 & 1 & & & & 0.5 \\
\hline 3 & 1 & 7 & & 1 & & 7 & 1 & & 0.666667 & & 1 & 0.24 & 1 & & & & \\
\hline 3 & 1 & & 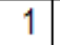 & 1 & & & & & 66667 & & 1 & 0.44 & 1 & & & & \\
\hline 3 & 1 & 1 & & 1 & 1 & & & & 1 & & 1 & 0.206897 & 1 & 0.8 & 0.5 & & 0.75 \\
\hline 3 & 1 & 1 & 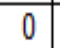 & 1 & 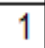 & 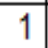 & 0 & & 666667 & & 1 & 0.31 & 1 & 0. & 1 & & 0.75 \\
\hline 3 & 1 & & & 1 & & & & & .666667 & & 1 & 0.51 & 1 & 0.2 & & & 0.75 \\
\hline 3 & 1 & 1 & 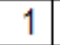 & 1 & & & & & 333 & & 5 & 0.37 & 1 & & & & 0.75 \\
\hline 3 & 1 & 1 & 1 & 1 & 1 & 1 & & 0.5 & 0.666667 & & 1 & 0.37931 & 1 & 0 & 1 & & 1 \\
\hline 3 & 1 & 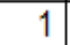 & & 1 & 1 & 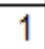 & & & & & 1 & 0.586 & 1 & 0.2 & 0.5 & & 0.75 \\
\hline 3 & 1 & & & 1 & & & & & 666667 & & 1 & & 1 & & 1 & & 0.5 \\
\hline 3 & 1 & 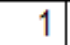 & & 1 & 1 & & & & & & 1 & 0.206897 & 0 & & & & 0.5 \\
\hline 3 & 1 & 7 & & 1 & 1 & ( & & & 333333 & & 1 & 0.58 & 1 & 0.2 & & & 0.5 \\
\hline 3 & 1 & 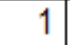 & 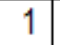 & 1 & 1 & & t & & 0.666667 & & 1 & 0.34 & 1 & & 1 & & 0.75 \\
\hline 3 & 1 & & & 1 & 1 & & 0 & 0. & 0.333333 & & 1 & 0.24 & 1 & 0.6 & 1 & & 0.75 \\
\hline 3 & 1 & & 1 & 1 & 1 & & & & 0.666667 & & 1 & 0.344828 & 1 & & 1 & & \\
\hline 3 & 1 & & 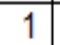 & 1 & 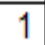 & 0 & & & & & 1 & 0.206897 & 0 & & 1 & & 0.5 \\
\hline 3 & 1 & & 1 & 1 & 0 & c & 0 & 0.5 & 666667 & & 1 & 0.48 & 1 & \begin{tabular}{|l|l|} 
& \\
\end{tabular} & 1 & 1 & 0.75 \\
\hline 3 & 1 & & 1 & 1 & 1 & 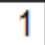 & & & 0.666667 & & 1 & 0.310345 & 1 & 0.2 & 1 & & 0.5 \\
\hline 3 & 1 & 1 & 1 & 1 & 0 & 1 & & & 0.333333 & & 1 & 0.448276 & 1 & \begin{tabular}{|l|} 
\\
\end{tabular} & 1 & & 0.5 \\
\hline 3 & 1 & 1 & & 1 & 1 & 0 & & 0.5 & 666667 & & 1 & & 1 & 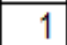 & 4 & 1 & 0.75 \\
\hline 3 & 1 & 1 & 1 & 1 & 1 & 0 & 0 & & 0.666667 & & 1 & 345 & 1 & 0.2 & 1 & & \\
\hline 3 & 1 & & 1 & 1 & 1 & 0 & 1 & 0 & 0 & & 1 & 0.482759 & 1 & 0.4 & 1 & 1 & 0.75 \\
\hline 3 & 1 & & & 1 & 1 & 1 & & & 1 & & & & 1 & 0 & 0.5 & & 0.5 \\
\hline 3 & 1 & 1 & 1 & 1 & 1 & 1 & 1 & 0.5 & 333333 & & 1 & 0.068966 & 1 & 0 & 1 & 1 & 1 \\
\hline 3 & 1 & 1 & 1 & 1 & 1 & 1 & 0 & & 666667 & & 1 & 0.31 & 1 & 0 & 1 & 1 & 0.75 \\
\hline 3 & 1 & 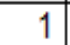 & 1 & 1 & 0 & 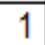 & 1 & 0 & 0.666667 & & 0.5 & 0.344828 & 1 & & 1 & & 0.5 \\
\hline & 1 & & & & & & 1 & & 0.666667 & & & 0.586207 & 1 & & 1 & 1 & 0.75 \\
\hline
\end{tabular}




\begin{tabular}{|c|c|c|c|c|c|c|c|c|c|c|c|c|c|c|c|c|c|}
\hline 3 & 1 & 0.5 & 1 & & & 1 & 1 & 0.5 & 0.666667 & & 0.5 & 0.62069 & 1 & 0 & 1 & 1 & 0.75 \\
\hline 3 & 1 & 1 & 1 & 1 & 1 & 0 & 1 & 0.5 & 0 & 0 & 0.5 & 0.551724 & 1 & 0 & 1 & 1 & \\
\hline 3 & 1 & 1 & 1 & 1 & 1 & 1 & 1 & 0.5 & 0.666667 & 0 & 1 & 0.482759 & 1 & 0.4 & 1 & 1 & 0.5 \\
\hline 3 & 1 & 0 & & c & 1 & 0 & 1 & 0.5 & 0 & & 1 & 0.241379 & 1 & 1 & 1 & & 0.5 \\
\hline 3 & 1 & 0.5 & 1 & 0 & 1 & 0 & 1 & 0.5 & 0.666667 & & 1 & 0.344828 & 1 & 0 & 1 & 1 & 0.5 \\
\hline 3 & 1 & 0 & 1 & 1 & 1 & 1 & 1 & 0 & 0.333333 & 1 & 1 & 0.241379 & 1 & 0.2 & 1 & 1 & 0.5 \\
\hline 3 & 1 & 1 & 1 & 1 & 1 & 0 & 0 & 0.5 & 0.666667 & 0 & 1 & 0.206897 & 1 & 0.2 & 1 & 1 & 0.75 \\
\hline 3 & 1 & 1 & 1 & 1 & 1 & 1 & 1 & 0 & 0.333333 & & 1 & 0.37931 & 1 & 1 & 1 & 1 & 0.75 \\
\hline 3 & 1 & 1 & 1 & 0 & 1 & 1 & 1 & 0.5 & 0.666667 & & 1 & 0.482759 & 1 & 0 & 1 & 1 & 0.5 \\
\hline 3 & 1 & 1 & 1 & 0 & 1 & 1 & 1 & 0 & 0.666667 & 0 & 1 & 0.344828 & 1 & 0 & 1 & 0 & 0.5 \\
\hline 1 & 0 & 1 & 1 & 1 & 1 & 1 & 0 & 0.5 & 0.666667 & 0 & 1 & 0.37931 & 1 & 0.4 & 1 & 0 & 0.75 \\
\hline 1 & 0 & 0 & 1 & 1 & 1 & 1 & 0 & 0 & 0.333333 & & 1 & 0.758621 & 1 & 0.4 & 1 & 1 & 0.5 \\
\hline l & 0 & 1 & 1 & 0 & 1 & 1 & 1 & 0 & 0.333333 & & 1 & 0.655172 & 1 & 0.4 & 1 & 1 & 0.75 \\
\hline 1 & 0 & 1 & 1 & 1 & 1 & 1 & 1 & 0.5 & 0 & 0 & 1 & 0.793103 & 1 & 0 & 1 & 0 & 0.5 \\
\hline l & 0 & 1 & 1 & 1 & 0 & 1 & 1 & 0.5 & 0.333333 & 0 & 1 & 0.344828 & 1 & 0.6 & 1 & 1 & 0.75 \\
\hline l & 0 & 1 & 1 & 0 & 0 & 1 & 0.5 & 0.5 & 0.666667 & & 1 & 0.655172 & 1 & 0.4 & 1 & 1 & 0.5 \\
\hline 1 & 0 & 1 & 1 & 1 & 1 & 1 & 0.5 & 1 & 0 & & 1 & 0.344828 & 0 & 1 & 1 & 1 & 0.25 \\
\hline 1 & 0 & 1 & 1 & 1 & 1 & 1 & 1 & 0 & 0.333333 & 0 & 1 & 0.275862 & 0 & \begin{tabular}{|l|l|}
0.8 \\
\end{tabular} & 1 & 1 & 0.5 \\
\hline 1 & 0 & 0 & 1 & 1 & 1 & 1 & 1 & 1 & 0.333333 & 0 & 1 & 0.37931 & 1 & 0.4 & 1 & 1 & 0 \\
\hline 1 & 0 & 1 & 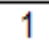 & 1 & 1 & 1 & 1 & 1 & 0.333333 & 0 & 1 & 0.413793 & 1 & 1 & 1 & 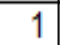 & 0.5 \\
\hline 1 & 0 & 1 & 1 & 1 & 1 & 1 & 1 & 1 & 0.333333 & & 1 & 0.275862 & 1 & \begin{tabular}{|l|l}
0.4 \\
\end{tabular} & 1 & 1 & 0.25 \\
\hline 1 & 0 & 1 & 1 & 1 & 1 & 1 & 1 & 0.5 & 0.666667 & 0 & 1 & 0.275862 & 1 & 0 & 1 & 1 & 0.75 \\
\hline 1 & 0 & 1 & 0 & 1 & 1 & 1 & 0 & & 0.333333 & 0 & 1 & 0.724138 & 1 & 0.4 & 1 & 1 & 0.75 \\
\hline l & 0 & 1 & 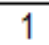 & 1 & 1 & 1 & 1 & 0.5 & 0.666667 & & 1 & 0.862069 & 1 & 0.2 & 1 & 1 & 0.75 \\
\hline 1 & 0 & 1 & 1 & 1 & 1 & 1 & 1 & 1 & 0.666667 & & 1 & 0.275862 & 1 & 0 & 1 & 1 & 0.25 \\
\hline 1 & 0 & 1 & 1 & 1 & 1 & 1 & 0 & 1 & 0.333333 & 0 & 1 & 0.448276 & 1 & 0.6 & 1 & 1 & 0.25 \\
\hline 1 & 0 & 0 & 1 & 1 & 1 & 1 & 1 & 1 & 0.666667 & & 1 & 0.827586 & 1 & 0.2 & 1 & 1 & 0.5 \\
\hline 1 & 0 & 1 & 1 & 1 & 1 & 1 & 0 & 0.5 & 0.666667 & & 1 & 0.275862 & 1 & 0.2 & 1 & 1 & 0.75 \\
\hline 1 & 0 & 1 & 1 & 0 & 1 & 1 & 1 & 1 & 0.333333 & & 1 & 0.586207 & 1 & 0.4 & 1 & 1 & 0.5 \\
\hline 1 & 0 & 1 & 1 & 1 & 1 & 1 & 0 & 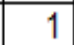 & 0.333333 & 0 & 1 & 0.37931 & 1 & \begin{tabular}{|l|}
0.2 \\
\end{tabular} & 1 & 1 & 0.25 \\
\hline 1 & 0 & 0 & 1 & 1 & 1 & 1 & . & 0 & 0.666667 & 0 & 0.5 & 0.206897 & 1 & 0 & 1 & 0 & 0.25 \\
\hline 1 & 0 & 1 & 0 & 1 & 1 & 1 & 1 & 1 & 0.666667 & 0 & 1 & 0.275862 & 1 & 0.4 & 1 & 1 & 0.5 \\
\hline 1 & 0 & 1 & 1 & 1 & 1 & 1 & 0 & 1 & 0.333333 & 0 & 1 & 0.206897 & 1 & 0.4 & 1 & 1 & 0.5 \\
\hline 1 & 0 & 1 & 1 & 1 & 1 & 1 & & & 1 & 0 & 1 & 0.206897 & 0 & 1 & 0.5 & 1 & 0.5 \\
\hline l & 0 & 1 & 1 & 1 & 1 & 1 & 1 & 1 & 1 & 0 & 1 & 0.206897 & 0 & 1 & 0.5 & 1 & 0.25 \\
\hline 1 & 0 & 0 & 1 & 0 & 1 & 1 & 1 & 1 & 0.666667 & 1 & 1 & 0.551724 & 1 & 1 & 1 & 1 & 0 \\
\hline l & 0 & 1 & 1 & 1 & 1 & 1 & 1 & 0.5 & 0.333333 & 0 & 1 & 0.310345 & 1 & 0.6 & 1 & 1 & 0.5 \\
\hline 1 & 0 & 1 & 0 & 1 & 1 & 1 & 0 & & 0.333333 & 0 & 1 & 0.310345 & 1 & 0.6 & 1 & 1 & 0 \\
\hline 1 & 0 & 1 & 1 & 1 & 1 & 1 & 0 & 0.5 & 0.666667 & 1 & 1 & 0.517241 & 1 & \begin{tabular}{|l|} 
\\
\end{tabular} & 1 & 1 & 0.5 \\
\hline 1 & 0 & 1 & 1 & 1 & 1 & 1 & 0 & 0 & 0.666667 & 1 & 1 & 0.62069 & 1 & 0 & 1 & 1 & 0.5 \\
\hline l & 0 & 1 & 1 & 1 & 1 & 1 & 1 & 0.5 & 0.333333 & 1 & 1 & 0.37931 & 1 & 0 & 1 & 1 & 0.5 \\
\hline 1 & 0 & 1 & 0 & 1 & 1 & 1 & 1 & 1 & 0.666667 & 0 & 1 & 0.517241 & 1 & 0.6 & 1 & 1 & 0.25 \\
\hline 1 & 0 & 1 & 1 & 0 & 1 & 1 & 0 & 1 & 0.333333 & 0 & 1 & 0.344828 & 1 & 0.6 & 1 & 1 & 0 \\
\hline 1 & 0 & 1 & 1 & 1 & 1 & 0 & 1 & 1 & 0.666667 & 0 & 1 & 0.275862 & 1 & 0 & 1 & 1 & 0.25 \\
\hline 1 & 0 & 1 & 1 & 1 & 1 & 1 & 1 & 1 & 1 & 0 & 1 & 0.206897 & 1 & \begin{tabular}{|l|} 
\\
\end{tabular} & 0.5 & 1 & 0.25 \\
\hline
\end{tabular}




\begin{tabular}{|c|c|c|c|c|c|c|c|c|c|c|c|c|c|c|c|c|c|}
\hline 1 & c & 1 & 1 & 1 & & 1 & 1 & 1 & 0.666667 & 1 & 1 & 0.689655 & 1 & 0 & 1 & 1 & 0.25 \\
\hline 1 & 0 & 1 & 1 & 1 & 1 & 0 & 1 & 0 & 0.666667 & 0 & 1 & 0.862069 & 1 & 0.2 & 1 & 1 & 0.5 \\
\hline 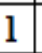 & 0 & 1 & 0 & $\sqrt{1}$ & 1 & 1 & 4 & 0 & 1 & & 1 & 0.206897 & 1 & 0.6 & 1 & & \\
\hline & & & 1 & & & & & & 666667 & & 1 & 0.344828 & 1 & 0.4 & & & 0.5 \\
\hline 1 & & 1 & 1 & 1 & 1 & 0 & & 0 & 333333 & & 1 & 0.275862 & 1 & 0.4 & 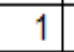 & 1 & 0.75 \\
\hline 1 & 0 & 1 & 0 & 1 & 1 & & 1 & 0.5 & 0.333333 & & 1 & 0.413793 & 1 & 0.2 & 1 & 1 & 0.75 \\
\hline 1 & 0 & 1 & 0 & 1 & & & 1 & 5 & 1 & & 1 & 0.655172 & 1 & 0 & & & 0.5 \\
\hline l & & 1 & 1 & 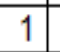 & & & & & 666667 & & 1 & 0.206897 & 1 & 0.2 & & & 0.5 \\
\hline 1 & 0 & 1 & 1 & 1 & 1 & 1 & 1 & 0.5 & 666667 & 0 & 1 & 0.655172 & 1 & 0.2 & 1 & & 0.5 \\
\hline 1 & 0 & 1 & 1 & 1 & 1 & & 0 & 0 & 0.666667 & & 1 & 0.344828 & 1 & 0.2 & 1 & & 0.5 \\
\hline 1 & 0 & 1 & 1 & 1 & & & & 1 & 0.666667 & & 1 & 0.344828 & 1 & 0 & & & 0.5 \\
\hline 1 & & 1 & 1 & 1 & & 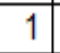 & 1 & 0.5 & 0.333333 & & 1 & 0.310345 & 1 & 0.4 & 1 & 1 & 0.5 \\
\hline l & 0 & 1 & 1 & 1 & & & & 1 & 333333 & & 1 & 0.241379 & 1 & 1 & 1 & & 0 \\
\hline 1 & 0 & 1 & 1 & 1 & 1 & & 1 & 0.5 & 0.666667 & & 1 & 0.413793 & 1 & 0 & 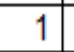 & & 0.5 \\
\hline 1 & 0 & 1 & 0.5 & 1 & 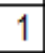 & 1 & & 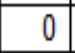 & 0.333333 & & 1 & 0.551724 & 1 & 0.8 & 1 & & \\
\hline 1 & 0 & 1 & ( & 1 & & 7 & 0 & & 0.666667 & 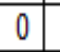 & 1 & 0.37931 & 1 & 0.4 & & 1 & 0.75 \\
\hline 1 & & 1 & 1 & 1 & & & & & 0.666667 & & 1 & 0.275862 & 1 & 0 & 1 & & 0.5 \\
\hline 1 & 0 & 1 & 1 & 1 & & & 1 & & 0.333333 & 0 & 1 & 0.62069 & 1 & 0.6 & 1 & & 0.5 \\
\hline 1 & 0 & 0 & 1 & 1 & 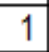 & 1 & & & 333333 & 0 & 0 & 0.344828 & 1 & 0.4 & 1 & 1 & 0.25 \\
\hline 1 & 0 & & 1 & 1 & & 1 & & & 666667 & 0 & 1 & 0.413793 & 1 & 0 & & & 0.5 \\
\hline 1 & 0 & & 1 & 1 & & & & 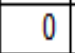 & 0.666667 & & 1 & 0.448276 & 1 & 0.2 & 1 & & 0.5 \\
\hline 1 & c & 1 & 1 & 1 & 1 & & & 1 & 0.666667 & & 1 & 0.689655 & 1 & 0.6 & 1 & 1 & 0.25 \\
\hline l & 0 & 1 & 0.5 & 1 & & 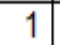 & & 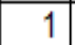 & 0 & & 1 & 0.482759 & 1 & 0.8 & & & \\
\hline 1 & & & 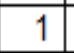 & 1 & & & 0 & & 666667 & & 1 & 0.344828 & & 0 & & & 0.5 \\
\hline 1 & & & ( & 1 & & & & & 0 & & 1 & 0.206897 & 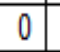 & 1 & 1 & & \\
\hline 1 & 0 & & 1 & 1 & 1 & & & 1 & 333333 & & 1 & 0.206897 & 1 & 0.2 & 1 & & 0 \\
\hline l & 0 & & 1 & 1 & & & & & 666667 & 0 & 1 & 0.37931 & 1 & 0 & & & 0.5 \\
\hline 1 & 0 & & 7 & 1 & & 1 & 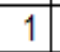 & 0. & 333333 & 1 & 1 & 0.172414 & 1 & 0.2 & 1 & 1 & 0.75 \\
\hline 1 & 0 & & 1 & 1 & 1 & & & 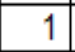 & 0.666667 & & 1 & 0.275862 & 1 & 0 & 1 & & 0.5 \\
\hline 1 & 0 & & 1 & 1 & 1 & & & 1 & 333333 & & 1 & 0.241379 & 1 & 0.8 & 1 & 1 & 0 \\
\hline 1 & 0 & & 1 & 1 & & & & 1 & 333333 & 0 & 1 & 0.310345 & 1 & 0.4 & 1 & 1 & 0.25 \\
\hline 1 & 0 & & 1 & 1 & & 1 & & 1 & 0.333333 & & 1 & 0.275862 & 1 & 0.8 & 1 & & \\
\hline l & 0 & & 1 & 1 & 1 & & & & 0 & & 1 & 0.62069 & 1 & 0.8 & 1 & & \\
\hline 1 & 0 & 0 & 1 & 1 & 1 & & & & 666667 & 1 & 1 & 0.551724 & 1 & 0.6 & 1 & 1 & 0.25 \\
\hline 1 & 0 & & 1 & 1 & & 1 & & & 333333 & 0 & 1 & 0.62069 & 1 & 0.6 & 1 & 1 & 0.25 \\
\hline 1 & 0 & & 1 & 1 & 1 & 1 & 1 & & 0 & 0 & 1 & 0.62069 & 1 & 0.6 & 1 & 1 & 0.5 \\
\hline l & 0 & & 1 & 1 & 1 & 1 & 1 & & 666667 & 0 & 1 & 0.344828 & 1 & 0.4 & 1 & & 0.5 \\
\hline 1 & 0 & 1 & 1 & 1 & 1 & 1 & 1 & & 0.666667 & 0 & 1 & 0.344828 & 1 & 0 & 1 & 1 & 0.5 \\
\hline 1 & 0 & & 1 & 1 & 1 & 1 & 1 & 0. & 0 & 0 & 1 & 0.206897 & 0 & 1 & 1 & & 0.5 \\
\hline 1 & 0 & 0 & 1 & 1 & 0 & 1 & & 1 & 0.333333 & 0 & 1 & 0.310345 & 1 & 0.6 & 1 & 1 & 0.5 \\
\hline l & 0 & 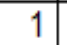 & 1 & 1 & 1 & 0 & & & 1 & 0 & 1 & 0.551724 & 1 & 0.4 & 1 & & 0.5 \\
\hline 1 & 0 & 1 & 0 & 1 & 1 & 1 & 1 & 1 & 1 & 0 & 1 & 0.724138 & 1 & 0.2 & 0.5 & 0 & 0 \\
\hline 1 & 0 & 1 & 1 & 1 & 1 & 1 & 1 & & 666667 & 0 & 1 & 0.689655 & 1 & 0 & 1 & 0 & 0.25 \\
\hline l & 0 & 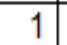 & 1 & 1 & 1 & 1 & 1 & 0.5 & 666667 & & 1 & 0.586207 & 1 & 0 & 1 & 1 & \\
\hline & 0 & & 1 & 1 & & & & & 0.333333 & 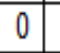 & 1 & 0.241379 & & 1 & 1 & 1 & 0.25 \\
\hline
\end{tabular}




\begin{tabular}{|c|c|c|c|c|c|c|c|c|c|c|c|c|c|c|c|c|c|}
\hline 1 & 0 & 1 & 1 & & & 1 & 1 & 1 & 0.333333 & 0 & 1 & 0.758621 & 1 & 0 & 1 & 1 & 0.25 \\
\hline 1 & 0 & 1 & 1 & 1 & 1 & 1 & 1 & 0.5 & 0.666667 & 0 & 1 & 0.241379 & 1 & 0 & 1 & 1 & 0.75 \\
\hline 1 & 0 & 1 & 1 & 1 & 1 & 1 & 0 & 1 & 0.333333 & 1 & 1 & 0.793103 & 1 & 0.4 & 1 & 1 & 0.25 \\
\hline 1 & 0 & 1 & 1 & 1 & 1 & 1 & 1 & 1 & 0.666667 & & 1 & 0.275862 & 1 & 0.2 & 1 & 1 & 0.25 \\
\hline 1 & 0 & 1 & 1 & 1 & 1 & 1 & 0 & 0.5 & 0.666667 & & 1 & 0.344828 & 1 & 0 & 1 & 1 & 0.5 \\
\hline 1 & 0 & 1 & 1 & 1 & 1 & 0 & 0.5 & 1 & 0.666667 & 0 & 1 & 0.62069 & 1 & 0.4 & 1 & 1 & 0.5 \\
\hline 2 & 0 & 1 & 1 & 1 & 1 & 1 & 1 & 1 & 0 & 0 & 1 & 0.344828 & 1 & 0.6 & 1 & 1 & 0.25 \\
\hline 2 & 0 & 1 & 1 & 1 & 1 & 1 & 1 & 0.5 & 0.666667 & 0 & 1 & 0.517241 & 1 & 0 & 1 & 1 & 0.5 \\
\hline 2 & 0 & 1 & 0 & 1 & 1 & 1 & 0 & 0 & 0.333333 & 1 & 1 & 0.344828 & 1 & 1 & 1 & 1 & 0.75 \\
\hline 2 & 0 & 1 & 1 & 1 & 1 & 1 & 1 & 0.5 & 0.666667 & 0 & 1 & 0.62069 & 1 & 0.4 & 1 & 1 & 0.75 \\
\hline 2 & 0 & 0 & 1 & 1 & 1 & 1 & 1 & 1 & 0 & 0 & 1 & 0.034483 & 0 & 1 & 1 & 1 & 0 \\
\hline 2 & 0 & 0 & 1 & 1 & 1 & 1 & 0 & 0 & 0.333333 & 1 & 1 & 0.275862 & 1 & 1 & 1 & 1 & 0.75 \\
\hline 2 & 0 & 1 & 1 & 1 & 0 & 0 & 1 & 0.5 & 0 & 0 & 1 & 0.413793 & 1 & 0.4 & 1 & 1 & 0.75 \\
\hline 2 & 0 & 0 & 0 & 1 & 1 & 1 & 0 & 0.5 & 0.333333 & 0 & 1 & 0.275862 & 1 & 0.4 & 1 & 1 & 0.75 \\
\hline 2 & 0 & 1 & 1 & 1 & 1 & 1 & 1 & 0.5 & 0.333333 & 0 & 1 & 0.724138 & 1 & 0 & 1 & 1 & 0.5 \\
\hline 2 & 0 & 1 & 1 & 1 & 1 & 1 & 0 & 1 & 0.333333 & 0 & 1 & 0.413793 & 1 & 0.4 & 1 & 1 & 0.25 \\
\hline 2 & 0 & 1 & 1 & 1 & 1 & 1 & 1 & 0 & 0.666667 & 0 & 1 & 0.275862 & 1 & 0 & 1 & 0 & 0.5 \\
\hline 2 & 0 & 1 & 0 & 1 & 1 & 1 & 1 & 0.5 & 0.666667 & 0 & 1 & 0.413793 & 1 & 0.4 & 1 & 1 & 1 \\
\hline 2 & 0 & 1 & 1 & 1 & 1 & 0 & 0 & 0.5 & 0.666667 & 0 & 1 & 0.689655 & 1 & 0.4 & 1 & 1 & 0.75 \\
\hline 2 & 0 & 1 & 1 & 1 & 1 & 1 & 1 & 1 & 0.666667 & 0 & 1 & 0.37931 & 1 & 0 & 1 & 1 & 0.25 \\
\hline 2 & 0 & 1 & 1 & 1 & 1 & 1 & 1 & 0.5 & 0.333333 & 0 & 1 & 0.551724 & 1 & \begin{tabular}{|l|l|}
0.4 \\
\end{tabular} & 1 & 1 & 0.5 \\
\hline 2 & 0 & 1 & 1 & 1 & 1 & 1 & 1 & 1 & 0.666667 & 0 & 1 & 0.37931 & 1 & 0 & 1 & 1 & 0.5 \\
\hline 2 & 0 & 0 & 1 & 1 & 1 & 1 & 1 & 1 & 0.666667 & 0 & 1 & 0.551724 & 1 & 0.4 & 1 & 1 & 0.25 \\
\hline 2 & 0 & 1 & 1 & 1 & 1 & 0 & 1 & 0.5 & 0 & 0 & 1 & 0.241379 & 1 & 0.2 & 1 & 1 & 0.5 \\
\hline 2 & 0 & 1 & 1 & 1 & 1 & 1 & 1 & 0.5 & 0.666667 & 0 & 1 & 0.689655 & 1 & 0 & 1 & 1 & 0.75 \\
\hline 2 & 0 & 0 & 1 & 1 & 1 & 1 & 1 & 0 & 0.666667 & 0 & 1 & 0.586207 & 1 & 0 & 1 & 0 & 0.5 \\
\hline 2 & 0 & 1 & 1 & 1 & 1 & 1 & 1 & 0 & 0.666667 & 0 & 1 & 0.206897 & 1 & 0 & 1 & 1 & 0.5 \\
\hline 2 & 0 & 1 & 1 & 1 & 1 & 1 & 1 & 0.5 & 0.666667 & 0 & 1 & 0.413793 & 1 & 0.4 & 1 & 1 & 0.5 \\
\hline 2 & 0 & 1 & 1 & 1 & 1 & 1 & 1 & 1 & 0.666667 & 0 & 1 & 0.482759 & 1 & 0.4 & 1 & 1 & 0.5 \\
\hline 2 & 0 & 1 & 1 & 1 & 1 & 1 & 1 & 0.5 & 0.666667 & 0 & 1 & 0.275862 & 1 & 0 & 1 & 1 & 0.75 \\
\hline 2 & 0 & 1 & 1 & 1 & 1 & 1 & 0 & 0.5 & 0.666667 & 1 & 1 & 0.724138 & 1 & 0.2 & 1 & 1 & 0.75 \\
\hline 2 & 0 & 1 & 1 & 1 & 1 & 1 & 1 & 0.5 & 0.666667 & 0 & 1 & 0.37931 & 1 & 0.2 & 1 & 1 & 0.5 \\
\hline 2 & 0 & 1 & 1 & 1 & 1 & 0 & 0 & 0.5 & 0.666667 & 1 & 1 & 0.344828 & 1 & 0.2 & 1 & 1 & 0.75 \\
\hline 2 & 0 & 1 & 1 & 1 & 1 & 1 & 1 & 0.5 & 0.333333 & 0 & 1 & 0.310345 & 1 & 1 & 1 & 1 & 0.75 \\
\hline 2 & 0 & 1 & 1 & 1 & 1 & 1 & 1 & 0.5 & 0.666667 & 0 & 1 & 1 & 1 & 0 & 1 & 0 & 0.75 \\
\hline 2 & 0 & 1 & 1 & 1 & 1 & 1 & 0 & 0.5 & 0.333333 & 0 & 1 & 0.275862 & 1 & 0.2 & 1 & 1 & 0.75 \\
\hline 2 & 0 & 1 & 1 & 1 & 1 & 1 & 1 & 0.5 & 0.666667 & 0 & 1 & 0.586207 & 1 & 0 & 1 & 1 & 1 \\
\hline 2 & 0 & 1 & 1 & 1 & 1 & 1 & 1 & 0.5 & 0.666667 & 0 & 1 & 0.793103 & 1 & 0.2 & 1 & 1 & 0.5 \\
\hline 2 & 0 & 1 & 1 & 1 & 1 & 1 & 1 & 0.5 & 0.333333 & 0 & 1 & 0.241379 & 0 & 1 & 1 & 1 & 0.5 \\
\hline 2 & 0 & 1 & 0 & 1 & 1 & 0 & 0 & 0.5 & 0.666667 & 0 & 1 & 0.310345 & 1 & 1 & 1 & 1 & 0.75 \\
\hline 2 & 0 & 1 & 0 & 1 & 1 & 1 & 0 & 0 & 0.666667 & 0 & 1 & 0.37931 & 1 & 0 & 1 & 1 & 0.75 \\
\hline 2 & 0 & 0.5 & 1 & 1 & 1 & 1 & 0 & 0.5 & 0.333333 & 0 & 1 & 0.310345 & 1 & 0.2 & 1 & 1 & 0.5 \\
\hline 2 & 0 & 1 & 1 & 1 & 1 & 1 & 0 & 0.5 & 0.333333 & 0 & 1 & 0.413793 & 1 & 0 & 1 & 1 & 0.75 \\
\hline 2 & 0 & 1 & 0.5 & 1 & 1 & 0 & 1 & 0 & 0.666667 & 0 & 1 & 0.344828 & 0 & 1 & 1 & 1 & 0.5 \\
\hline 2 & 0 & 1 & 1 & 1 & 1 & 1 & 1 & 0.5 & 0.666667 & 1 & 1 & 0.310345 & 1 & 0 & 1 & 1 & 0.5 \\
\hline
\end{tabular}




\begin{tabular}{|c|c|c|c|c|c|c|c|c|c|c|c|c|c|c|c|c|c|}
\hline & & & & & & & 1 & 0.5 & 333 & & 1 & 0.310345 & 1 & 0.8 & 1 & 1 & 0.5 \\
\hline 2 & c & 1 & 1 & 1 & 1 & & 0 & 0.5 & 33333 & & 1 & 0.206897 & 1 & 1 & & 1 & \\
\hline & & & & & & & 1 & 5 & 666667 & & & 0.37931 & & & & & 0.75 \\
\hline 2 & & & & & & & 1 & 0.5 & 0 & & 1 & 0.103448 & 0 & & & & 0.5 \\
\hline & & & & & & & 1 & & 333333 & & 1 & 0.655172 & & & & & 0.5 \\
\hline 2 & 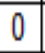 & & & 1 & & & 0 & & 333 & & 1 & 0.310345 & 1 & & & & 0.5 \\
\hline 2 & & 1 & & 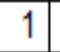 & 1 & 1 & 0.5 & 0.5 & 666667 & & 1 & 0.275862 & 1 & 0.4 & & & \\
\hline 2 & & & & & 1 & & & 0 & 333333 & & 1 & 0.206897 & 0 & & & & 0.5 \\
\hline 2 & & 1 & 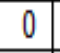 & 1 & 1 & & & 0 & 666667 & & 1 & 0.310345 & 0 & & & 7 & 0.25 \\
\hline 2 & & & & & 1 & & 0 & 0.5 & .666667 & & 1 & 0.344828 & 1 & 0.4 & & & 0.5 \\
\hline 2 & c & & & 1 & 1 & & & 0 & 33 & & 0.5 & 0.413793 & 1 & & & & 0.75 \\
\hline 2 & ( & 1 & 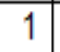 & 1 & 1 & 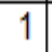 & 0 & 0 & 0.333333 & & 1 & 0.517241 & 1 & 0.2 & & 1 & 0.25 \\
\hline 2 & ( & & & 1 & 1 & & & 0.5 & 333 & & 1 & 0.413793 & 1 & 0. & & 1 & 0.75 \\
\hline 2 & 0 & & & 1 & 1 & & 0 & 0.5 & 666667 & & 1 & 0.448276 & 1 & 0.2 & & & 0.5 \\
\hline 2 & & & & & 1 & & & & 666667 & & 1 & 0.275862 & 1 & 0.4 & & & 0.5 \\
\hline 2 & C & & & 1 & 1 & & 0 & 0.5 & 666667 & & 1 & 0.344828 & 1 & 0 & & 1 & 0.75 \\
\hline 2 & c & & & 1 & 1 & & & & ).333333 & & 1 & 0.310345 & 1 & & & 1 & 0.25 \\
\hline 2 & c & & 1 & 1 & 1 & & & 0 & 666667 & & 1 & 0.413793 & 1 & 0.4 & & 1 & 0.5 \\
\hline 2 & ( & & & 1 & 1 & 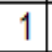 & & & .666667 & & 1 & 0.448276 & 1 & & & 1 & 0.25 \\
\hline 2 & ( & & 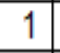 & 1 & 0 & 1 & & & 333333 & & 1 & 0.517241 & 1 & 0.6 & & & 0.5 \\
\hline 2 & ( & & & 1 & 1 & & & 0.5 & 0.6666667 & & 1 & 0.310345 & 1 & & & 1 & 0.5 \\
\hline 2 & 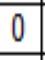 & & & 1 & 1 & & & & & & 1 & 0.482759 & 1 & & & & 0.5 \\
\hline 2 & ( & & & 1 & 1 & & & & 333333 & & 1 & 0.448276 & 1 & 0.4 & & 1 & 0.75 \\
\hline 2 & ( & & & 1 & 1 & & & 0.5 & 666667 & & 1 & 0.517241 & 1 & 0 & & 1 & 0.75 \\
\hline 2 & ( & & & 1 & 1 & & & & .666667 & & 1 & 0.448276 & 1 & 0.2 & & 1 & 0.75 \\
\hline 2 & c & 1 & & 1 & 1 & & & & 666667 & & 1 & 0.655172 & 1 & & & 1 & 0.5 \\
\hline 2 & ( & 1 & 1 & 1 & 1 & 1 & & 0 & 0.666667 & & 1 & 0.758621 & 1 & & & 1 & 0.25 \\
\hline 2 & 0 & & & 1 & 1 & & 1 & 0.5 & 666667 & & 1 & 0.827586 & 1 & 0.8 & & 1 & 0.75 \\
\hline 2 & 0 & 1 & 1 & 1 & 1 & 1 & & 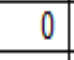 & .333333 & & 1 & 0.344828 & 1 & & 1 & 1 & 0.75 \\
\hline 2 & 0 & 1 & & 1 & 1 & 0 & & 0.5 & .666667 & & 1 & 0.310345 & 1 & & & 1 & 0.5 \\
\hline 2 & 0 & 1 & 0 & 1 & 1 & 1 & & 1 & 0.333333 & & 1 & 0.344828 & 0 & & 1 & 1 & 0.25 \\
\hline 2 & 0 & 1 & 1 & 1 & 1 & 1 & & & 0.666667 & & 1 & 0.551724 & 1 & 0.2 & & 1 & 0.5 \\
\hline & 0 & 1 & 1 & 1 & 1 & 1 & 0 & 1 & 666667 & 0 & 1 & 0.206897 & 1 & 0.2 & 1 & 1 & 0.5 \\
\hline
\end{tabular}

